\title{
Nonlinear wave-activity conservation laws and Hamiltonian structure for the two- dimensional anelastic equations
}

Article

Published Version

Scinocca, J. F. and Shepherd, T. G. (1992) Nonlinear waveactivity conservation laws and Hamiltonian structure for the two-dimensional anelastic equations. Journal of the Atmospheric Sciences, 49 (1). pp. 5-28. ISSN 1520-0469 doi: https://doi.org/10.1175/15200469(1992)049<0005:NWACLA>2.0.CO;2 Available at https://centaur.reading.ac.uk/32971/

It is advisable to refer to the publisher's version if you intend to cite from the work. See Guidance on citing.

Published version at: http://dx.doi.org/10.1175/1520-0469(1992)049<0005:NWACLA>2.0.CO;2

To link to this article DOI: http://dx.doi.org/10.1175/1520-

0469(1992)049<0005:NWACLA>2.0.CO;2

Publisher: American Meteorological Society

All outputs in CentAUR are protected by Intellectual Property Rights law, including copyright law. Copyright and IPR is retained by the creators or other copyright holders. Terms and conditions for use of this material are defined in the End User Agreement. 


\section{CentAUR}

Central Archive at the University of Reading

Reading's research outputs online 


\title{
Nonlinear Wave-Activity Conservation Laws and Hamiltonian Structure for the Two-Dimensional Anelastic Equations
}

\author{
J. F. SCINOCCA AND T. G. SHEPHERD \\ Department of Physics, University of Toronto, Toronto, Canada \\ (Manuscript received 10 January 1991, in final form 15 May 1991)
}

\begin{abstract}
Exact, finite-amplitude, local wave-activity conservation laws are derived for disturbances to steady flows in the context of the two-dimensional anelastic equations. The conservation laws are expressed entirely in terms of Eulerian quantities, and have the property that, in the limit of a small-amplitude, slowly varying, monochromatic wave train, the wave-activity density $A$ and flux $\mathbf{F}$, when averaged over phase, satisfy $\mathbf{F}=c_{g} A$ where $c_{g}$ is the group velocity of the waves. For nonparallel steady flows, the only conserved wave activity is a form of disturbance pseudoenergy; when the steady flow is parallel, there is in addition a conservation law for the disturbance pseudomomentum.

The above results are obtained not only for isentropic background states (which give the so-called "deep form" of the anelastic equations), but also for arbitrary background potential-temperature profiles $\theta_{0}(z)$ so long as the variation in $\theta_{0}(z)$ over the depth of the fluid is small compared with $\theta_{0}$ itself. The Hamiltonian structure of the equations is established in both cases, and its symmetry properties discussed. An expression for available potential energy is also derived that, for the case of a stably stratified background state (i.e., $d \theta_{0} / d z>0$ ), is locally positive definite; the expression is valid for fully three-dimensional flow.

The counterparts to these results for the two-dimensional Boussinesq equations are also noted.
\end{abstract}

\section{Introduction}

In many fluid dynamical problems it is advantageous to consider the fluid state as a disturbance to some specified (and comparatively simple) basic state. Such a decomposition is implicit in any linearized theory, of course, but it may nevertheless be useful under more general circumstances. (Typically one would consider basic states having particular symmetries, steady flows and parallel flows as being especially common examples.) Having chosen to represent the problem in this fashion, wave-activity conservation laws frequently turn out to play a central role in the discussion (see, e.g., Bretherton 1971; Hoskins 1983; Held and Hoskins 1985; Andrews et al. 1987).

A wave activity may be defined to be a conserved disturbance quantity that is quadratic (or of higher order) in the disturbance fields in the small-amplitude limit. An important consequence of this is that the wave activity may be evaluated correct to leading order from a solution calculated on the basis of linearized theory, and so on to higher orders in disturbance amplitude; this property, together with the fact that it satisfies a conservation law, facilitates the construction of self-consistent theories. By contrast, the wave energy

Corresponding author address: Dr. T. G. Shepherd, Department of Physics, University of Toronto, 60 St. George St., Toronto, Ontario, Canada M5S 1A7. (i.e., the energy of the disturbance in a frame of reference moving with the basic flow) is frequently quadratic but is not conserved, while the disturbance energy (i.e., the difference between the total energy and the energy of the basic state) is conserved but is not quadratic, and, thus, is not calculable even to leading order from linearized theory. By a conservation law, we mean a relation of the form

$$
\frac{\partial A}{\partial t}+\nabla \cdot \mathbf{F}=0
$$

where $A$ is the density of wave activity and $\mathbf{F}$ its flux.

Until recently, wave-activity conservation laws have been of one of three sorts. Either they have been restricted to small-amplitude (though possibly weakly nonlinear) disturbances under slowly varying, WKBlike conditions (e.g., Garrett 1968; Bretherton 1969; Young and Rhines 1980), in which case the conservation laws can be explicitly linked to symmetry properties of the system (Whitham 1965; Bretherton and Garrett 1968; Bretherton 1971); or they have been derived by direct manipulation of the linearized equations of motion (e.g., Andrews and McIntyre 1976, 1978a; Andrews 1983); or they have been finite amplitude but expressed in terms of particle displacements (Andrews and McIntyre 1978b; Grimshaw 1984). What one really desires for practical applications, however, is a systematic method of deriving finite-amplitude wave-activity conservation laws that are expressed in 
terms of Eulerian variables and do not require WKB assumptions.

It has recently become apparent (McIntyre and Shepherd 1987; see also Shepherd 1990, section 5 ) that the Hamiltonian formalism provides a natural way of doing just that. Indeed, even if one seeks only a smallamplitude result, the most direct way of deducing it turns out to be by appealing to the Hamiltonian structure of the finite-amplitude problem, and then taking the appropriate small-amplitude limit of the resulting wave-activity conservation law. In a recent application of the method, Haynes (1988) was able to systematically derive finite-amplitude wave-activity conservation laws for the hydrostatic primitive equations. (Haynes also showed that the results could always be generalized to forced dissipative systems, which may be important for applications.)

In this paper, finite-amplitude wave-activity conservation laws are derived for the two-dimensional anelastic equations. Both pseudoenergy and pseudomomentum conservation laws of the form (1.1) are obtained for disturbances to steady basic flows; in the former case the basic flow may be nonparallel, while in the latter it must be independent of the downstream coordinate $x$. The corresponding forms of the conservation laws are also recorded for the special case of the Boussinesq equations. An important feature of the forms derived here is that in the WKB limit, wherein the disturbance consists of a small-amplitude, monochromatic wave train, and the basic state is varying slowly over the scale of the disturbance, we obtain the physically sensible relation $\langle\mathbf{F}\rangle=\mathbf{c}_{g}\langle A\rangle$ (where the angle brackets denote an average over the phase of the waves). This condition ensures consistency between the wave-activity densities and fluxes derived here (which are valid even under non-WKB conditions), and those that would be obtained under a conventional small-amplitude WKB derivation. It may be noted that the satisfaction of this condition for the two-dimensional anelastic system, while in principle straightforward, turns out to require a considerable effort; in the case of the full primitive equations the situation would presumably be even more daunting.

An important intermediate step is the representation of the two-dimensional anelastic equations as a noncanonical Hamiltonian dynamical system. Such representation ensures an energetically consistent formulation, and enables an explicit link to be made between symmetries and conservation laws through Noether's theorem. The Hamiltonian formulation of the dynamics is provided both for the so-called "deep equations" of Ogura and Phillips (1962), where the background potential-temperature profile $\theta_{0}$ is taken to be constant, and for the modified set proposed by Lipps and Hemler (1982) when $\theta_{0}$ is a nonconstant function of $z$.

The plan of the paper is as follows. In section 2 we briefly review the Hamiltonian formalism by which the wave activity will be derived. The anelastic system is described in section 3. In section 4 the Hamiltonian structure of the anelastic system is presented and then exploited to derive Casimir and momentum invariants of the system. The methods described in section 2 are then applied to derive finite-amplitude expressions for pseudoenergy (section 5) and pseudomomentum (section 6), along with their corresponding fluxes, for disturbances to steady - and, for pseudomomentum, parallel-but otherwise arbitrary basic flows. The paper concludes with a discussion in section 7 .

\section{Hamiltonian theory}

In this section we shall briefly describe the Hamiltonian theory employed in this paper. The interested reader may refer to Goldstein (1980) for a thorough treatment of finite-dimensional canonical Hamiltonian dynamical systems. A mathematically rigorous discussion of the extension of this theory to noncanonical dynamical systems of infinite dimension may be found in Arnol'd (1978), Abraham and Marsden (1978), Marsden and Weinstein (1983), or Olver (1986). For the purposes of this paper, however, the most appropriate references would be the reviews by Benjamin (1984), Salmon (1988a), and Shepherd (1990).

\section{a. Symplectic representation}

The Hamiltonian formalism used in this paper requires that the governing system of equations be cast in the symplectic form

$$
U_{t}=J \frac{\delta \mathscr{H}}{\delta U} .
$$

In (2.1), $U$ is a column vector of the dependent field variables, while $\mathscr{H}$ is the total energy (or Hamiltonian) of the system and as such represents a functional (i.e., a function of the functions contained in $U$ ). The subscript $t$ refers to partial differentiation with respect to time. The term $\delta \mathscr{H} / \delta U$ is a column vector and refers to the variational or functional derivative of the functional $\mathscr{H}$ with respect to the functions contained in the vector $U$, and is defined for arbitrary functionals $\mathscr{F}$ by

$$
\mathcal{F}(U+\delta U)-\mathcal{F}(U)=\left(\frac{\delta \mathcal{F}}{\delta U}, \delta U\right)+\mathrm{O}\left(\delta U^{2}\right) .
$$

Here $(\cdot, \cdot)$ refers to the inner product

$$
(\mathfrak{F}, \mathcal{S}) \equiv \int \mathcal{F}_{i} \mathcal{S}_{i} d \mathbf{x}
$$

where the integral is over all physical space and a summation convention is implied by repeated indices. Finally, $J$ is a matrix operator that must satisfy

$$
\begin{aligned}
{[\mathcal{F}, a \mathcal{S}+b \mathcal{K}] } & =a[\mathcal{F}, \mathcal{S}]+b[\mathcal{F}, \mathcal{K}], \\
{[\mathcal{F}, \mathcal{S}] } & =-[\mathcal{S}, \mathcal{F}],
\end{aligned}
$$


$[\mathcal{F} \mathscr{S}, \mathcal{K}]=\mathscr{F}[\mathcal{S}, \mathcal{K}]+[\mathcal{F}, \mathcal{K}] \mathscr{S}$,

$[[\mathcal{F}, \mathscr{S}], \mathcal{K}]+[[\mathcal{S}, \mathcal{K}], \mathscr{F}]+[[\mathcal{K}, \mathscr{F}], \mathscr{S}]=0$,

where $[\cdot, \cdot]$ is the Poisson bracket

$$
[\mathcal{F}, \mathcal{S}] \equiv\left(\frac{\delta \mathcal{F}}{\delta U}, J \frac{\delta \mathscr{S}}{\delta U}\right),
$$

$\mathcal{F}, \mathcal{S}$, and $\mathcal{K}$ are functionals, and $a, b \in \mathcal{R}$. Of the four conditions on $J$, Jacobi's identity $(2.7)$ is by far the most arduous to verify.

\section{b. Integral invariants}

If it is possible to express a set of governing equations in the form (2.1), it then becomes a reasonably straightforward matter to identify the integral invariants of the system. By Noether's theorem, a functional $\mathcal{L}$ that satisfies

$$
J \frac{\delta \mathcal{L}}{\delta U}=-U_{\alpha}
$$

is time invariant if $\mathscr{H}$ is invariant under translations in $\alpha$. Thus, when $\alpha=t$, for example, invariance of the Hamiltonian under translations in time is seen to imply conservation of energy, since comparing (2.9) with (2.1) we have

$$
\mathcal{L}=-\mathscr{H} .
$$

If, on the other hand, $\alpha$ is the spatial coordinate $x$, say, then invariance of the Hamiltonian under translations in the $x$ direction implies conservation of the $x$-momentum $M$, where $M$ is defined by (2.9) with $\mathcal{L}=M$ and $\alpha=x$.

In addition to conserved quantities derived through Noether's theorem, one can identify Casimir invariants of the form

$$
\mathcal{e}=\int C(U) d \mathbf{x},
$$

where $C$ is some function of the dependent field variables. These functionals $\mathcal{C}$ are defined to be the solutions to

$$
J \frac{\delta \mathcal{C}}{\delta U}=0 .
$$

A comparison of (2.9) and (2.12) reveals that the Hamiltonian and generalized momentum integral invariants may be defined only to within a Casimir of the system.

For fluid dynamical problems, Casimir invariants typically represent explicit Lagrangian symmetries in the problem (e.g., particle relabeling) that are invisible in the Eulerian framework. As such, they convey valuable information that would otherwise be inaccessible in the Eulerian representation of the problem. Ham- iltonian theory [i.e., expressing a system in the form (2.1)] provides a systematic method by which these important Casimir invariants may be derived. The constraint imposed by (2.12) is usually only of sufficient strength to specify the function $C$ in the most general terms. It is the arbitrary nature of the function $C$ that one may then exploit to derive finite-amplitude expressions for the pseudoenergy and pseudomomentum of a system.

\section{c. Wave-activity conservation laws}

A wave activity is defined to be a functional of a disturbance to some given basic state $\bar{U}$ that is both conserved (for conservative flow) and is of quadratic or higher order in disturbance amplitude $a$ in the limit $a \rightarrow 0$. The second condition ensures that the wave activity may be calculated to leading order solely from a linearized solution of the problem. Given the integral invariants, derived from the Hamiltonian structure of a system, it becomes a straightforward matter to construct finite-amplitude forms of wave activity possessing such qualities from either the energy (whenever $\bar{U}$ is independent of $t$ ) or the momentum (whenever $\bar{U}$ is independent of $x$ ) of the system.

In deriving the pseudoenergy (i.e., the wave activity associated with energy), we construct the quantity

$$
\mathcal{A}=\mathscr{H}(U)-\mathscr{H}(\bar{U})+\mathcal{C}(U)-\mathcal{C}(\bar{U})
$$

subject to the condition that the first variation of $\mathcal{A}$, $\delta \mathcal{A}$, vanish when $U=\bar{U}$. Now, given that

$$
\begin{aligned}
\left.\delta \mathcal{A}\right|_{U=\bar{U}} & =\left(\left.\frac{\delta \mathcal{A}}{\delta U}\right|_{U=\bar{U}}, \delta U\right) \\
& =\left(\left.\frac{\delta \mathscr{H}}{\delta U}\right|_{U=\bar{U}}+\left.\frac{\delta \mathcal{C}}{\delta U}\right|_{U=\bar{U}}, \delta U\right),
\end{aligned}
$$

an $\mathcal{A}$ may generally be constructed such that $\left.\delta \mathcal{A}\right|_{U=\bar{U}}=0$ if $\bar{U}$ is a steady solution of (2.1), since

$$
\bar{U}_{t}=\left.J \frac{\delta \mathscr{H}}{\delta U}\right|_{U=\bar{U}}=\left.0 \rightarrow \frac{\delta \mathscr{C}}{\delta U}\right|_{U=\bar{U}}=-\left.\frac{\delta \mathscr{C}}{\delta U}\right|_{U=\bar{U}}
$$

for some Casimir $\mathcal{C}$ (e.g., see Abarbanel et al. 1986).

Armed with the functional $\mathcal{A}$, which defines the global conservation of wave activity, we may now determine local conservation laws of the form (1.1). Since

$$
\frac{d \mathcal{A}}{d t}=\int \frac{\partial A}{\partial t} d \mathbf{x}=0,
$$

it is always possible to write the temporal variation of wave-activity density $A$ in the flux form

$$
\frac{\partial A}{\partial t}=-\nabla \cdot \mathbf{F} .
$$

It is obvious that the local flux of wave activity $F$ so defined is not uniquely determined. In particular, one 
may add any vector field with zero divergence to $\mathbf{F}$, or even the time derivative of any vector field so long as the divergence of the same field is removed from $A$ (since this will not affect $\mathcal{A}$ ). The problem now reduces to determining appropriate forms of $A$ and $\mathbf{F}$. One may eliminate a great deal of this arbitrariness by imposing the constraint that the usual WKB result

$$
\langle\mathbf{F}\rangle=\mathbf{c}_{g}\langle A\rangle
$$

is recovered in the relevant limit of a small-amplitude, slowly varying, monochromatic wave train. In (2.16) the angle brackets denote an average over the phase of the disturbance fields.

For the case of pseudomomentum, one merely employs the generalized momentum $\mathcal{M}$ in place of the Hamiltonian $\mathscr{H}$ in (2.13). In sections 5 and 6 of this paper the reader will find a more detailed account of this method as we derive finite-amplitude wave activities for the anelastic and Boussinesq systems.

\section{The two-dimensional anelastic system}

If it is assumed that the pressure and density in the real atmosphere depart little from their distribution in an ideal atmosphere prescribed by a potential temperature varying slowly in the vertical (Batchelor 1953; Lipps and Hemler 1982), and that the fastest time scale upon which dynamically significant fluid motions occur is set by internal gravity waves (Ogura and Charney 1962 ), then the fundamental hydrodynamic equations for an ideal gas may be approximated by the anelastic equations. The term anelastic (Ogura and Phillips 1962) refers to the fact that the fastest physics, primarily associated with sound waves, is effectively filtered from the equations; this is achieved by neglecting the local time variation of density in the continuity equation. In this respect the anelastic system is quite similar to the set of equations obtained in the Boussinesq approximation. The primary difference between these two approximations lies in the ability of the anelastic system to describe an atmosphere incorporating a background density profile that decreases strongly in the vertical. As a result, the Boussinesq system may be seen as a subset of the set of equations derivative of the anelastic approximation.

In this paper we will consider the most general form of the anelastic approximation. The system of equations that results from such approximation is

$$
\begin{gathered}
\frac{\partial \mathbf{v}}{\partial t}+(\mathbf{v} \cdot \nabla) \mathbf{v}=-c_{p} \theta_{0} \nabla \tilde{\pi}-c_{p} \frac{d \pi_{0}}{d z} \tilde{\theta} \hat{\mathbf{z}}, \\
\frac{\partial \tilde{\theta}}{\partial t}+(\mathbf{v} \cdot \nabla) \tilde{\theta}+w \frac{d \theta_{0}}{d z}=0, \\
\nabla \cdot\left(\rho_{0} \mathbf{v}\right)=0 .
\end{gathered}
$$

In this system the thermodynamic fields have been written

$$
\begin{gathered}
\theta(x, z, t)=\theta_{0}(z)+\tilde{\theta}(x, z, t), \\
\pi(x, z, t)=\pi_{0}(z)+\tilde{\pi}(x, z, t),
\end{gathered}
$$

where the zero subscript refers to the background state and the tilde variables represent (small) perturbations,

$$
\pi=\left(\frac{p}{p_{00}}\right)^{\kappa}
$$

is the Exner pressure, $\theta$ the potential temperature, $\kappa$ $=R / c_{p}$, and $p_{00}$ is the surface reference pressure. In deriving this system we have also assumed that the background thermodynamic fields are in hydrostatic balance. All other variables and notation in (3.1)-(3.6) have their conventional meaning.

If one employs the definition of potential temperature

$$
\theta=T \pi^{-1},
$$

assumes that the background is in hydrostatic balance

$$
c_{p} \theta_{0} \frac{d \pi_{0}}{d z}=-g
$$

and invokes the ideal gas law $p=\rho R T$, then all of the background thermodynamic fields are uniquely determined in terms of the profile $\theta_{0}(z)$.

\section{a. Energy conservation}

In order to apply Hamiltonian theory, we require a system of equations that is energetically closed. It is well known that if $\theta_{0}(z)=$ const the anelastic system thereby obtained, usually referred to as the "deep equations" of Ogura and Phillips (1962), is energetically consistent. If, however, $\theta_{0}$ is allowed to vary in the vertical, Wilhelmson and Ogura (1972) have shown that the system (3.1)-(3.3) does not conserve total energy. The resolution of this problem was first presented by Lipps and Hemler (1982). [See also Lipps $(1990)$.] If one assumes that $\theta_{0}$ varies slowly in the vertical, then to the same order of approximation, (3.1) may be replaced by

$$
\frac{\partial \mathbf{v}}{\partial t}+(\mathbf{v} \cdot \nabla) \mathbf{v}=-\nabla\left(c_{p} \theta_{0} \tilde{\pi}\right)-c_{p} \frac{d \pi_{0}}{d z} \tilde{\theta} \hat{\mathbf{z}}
$$

the only difference being that $\theta_{0}$ has been brought under the gradient operator in the first term on the righthand side of (3.1). The inner product of $\rho_{0} \mathrm{v}$ and (3.9) results in the kinetic energy equation

$$
\begin{array}{r}
\frac{\partial}{\partial t}\left(\frac{\rho_{0}}{2}|\mathbf{v}|^{2}\right)+\nabla \cdot\left[\rho_{0} \mathbf{v}\left(\frac{1}{2}|\mathbf{v}|^{2}+c_{p} \theta_{0} \tilde{\pi}\right)\right] \\
=-c_{p} \rho_{0} w \frac{d \pi_{0}}{d z} \tilde{\theta} .
\end{array}
$$


In a similar manner, if (3.2) is multiplied by $c_{p} \rho_{0} \pi_{0}$, one obtains the potential energy equation

$$
\begin{array}{r}
\frac{\partial}{\partial t}\left(c_{p} \rho_{0} \pi_{0} \tilde{\theta}\right)+\nabla \cdot\left[\rho_{0} \mathbf{v}\left(c_{p} \pi_{0}\left(\theta_{0}+\tilde{\theta}\right)+g z\right)\right] \\
=c_{p} \rho_{0} w \frac{d \pi_{0}}{d z} \tilde{\theta} .
\end{array}
$$

In the derivation of (3.10) and (3.11) we have used the continuity equation (3.3) and hydrostatic balance relation (3.8). The total energy of the system is then the sum of (3.10) and (3.11) integrated over all space. Assuming simple boundary conditions, the result is

$$
\frac{d}{d t} \iint\left(\frac{\rho_{0}}{2}|\mathbf{v}|^{2}+c_{p} \rho_{0} \pi_{0} \theta\right) d x d z=0
$$

Note that we have written the total $\theta$ rather than $\tilde{\theta}$ in the potential energy term in (3.12). This involves no loss of generality, since the two terms differ by a quantity that is constant in time. The conservation relation (3.12) suggests the Hamiltonian

$$
\mathscr{H}=\iint\left(\frac{\rho_{0}}{2}|\mathbf{v}|^{2}+c_{p} \rho_{0} \pi_{0} \theta\right) d x d z .
$$

Now, $(3.13 \mathrm{a})$ is linear in the perturbation potential temperature $\tilde{\theta}$, and the available potential energy $\iint c_{p} \rho_{0} \pi_{0} \tilde{\theta} d x d z$ is therefore not of definite sign. This makes interpretation of energy budgets less than satisfactory. A remedy for this situation is available, however, whenever the background state is stably stratified, namely, $d \pi_{0} / d \theta_{0}<0$. We first recall from section $2 \mathrm{~b}$ that the Hamiltonian is defined only to within a Casimir. It is evident from the thermodynamic equation (3.2) that integrals of the form

$$
e=\iint \rho_{0} C(\theta) d x d z
$$

are conserved in time for arbitrary functions $C$, and it will be seen in section $4 \mathrm{~b}$ that these represent Casimirs. Under stably stratified conditions $\pi_{0}$ and $\theta_{0}$ are both monotonic functions of $z$, and the resulting dependence $\pi_{0}\left(\theta_{0}\right)$ defines a monotonic function $\pi_{0}(\cdot)$. Then choosing

$$
C(\theta)=-c_{p} \int \pi_{0}(\theta) d \theta
$$

we may take as the Hamiltonian

$$
\begin{aligned}
\mathscr{H}(\mathbf{v}, \theta)-\mathscr{H}\left(0, \theta_{0}\right)+\mathcal{C}(\theta)-\mathcal{C}\left(\theta_{0}\right) \\
=\iint\left(\frac{\rho_{0}}{2}|\mathbf{v}|^{2}-c_{p} \rho_{0} \int_{0}^{\tilde{\theta}}\left[\pi_{0}\left(\theta_{0}+\eta\right)\right.\right. \\
\left.\left.\quad-\pi_{0}\left(\theta_{0}\right)\right] d \eta\right) d x d z
\end{aligned}
$$

instead of (3.13a). This expression is of quadratic order in the perturbation fields, and is moreover positive definite under the aśsumption $d \pi_{0} / d \theta_{0}<0$. The "amount" of energy thus becomes a meaningful quantity, in the sense that the energy as defined according to (3.13b) may be used to provide a norm for the perturbation. The form (3.13b) is analogous to that derived by Holliday and McIntyre (1981) for a Boussinesq fluid, and by Andrews (1981) for a compressible fluid. It will be seen in section 5 that ( $3.13 \mathrm{~b}$ ) represents the pseudoenergy relative to the resting background state.

For the purpose of calculating energy conversions it may be useful to note the leading-order approximation to the available potential energy component of ( $3.13 b)$, which is

$$
\iint \frac{1}{2} \frac{\rho_{0} g^{2}}{N_{0}^{2} \theta_{0}^{2}} \tilde{\theta}^{2} d x d z,
$$

where $N_{0}$ is the Brunt-Väisälä frequency for the background state $\theta_{0}(z)$. It is worth emphasizing that the expressions $(3.13 \mathrm{~b}, \mathrm{c})$ are valid for fully three-dimensional flow.

\section{b. Steady-state and disturbance equations}

For two-dimensional flow, the equations (3.2), (3.3), and (3.9), which define the anelastic system, may be written in a more compact form. If we define the mass flux streamfunction $\psi$ by

$$
\rho_{0} \mathbf{v}=\hat{\mathbf{y}} \times \nabla \psi,
$$

so that

$$
u=\frac{1}{\rho_{0}} \frac{\partial \psi}{\partial z}, \quad w=-\frac{1}{\rho_{0}} \frac{\partial \psi}{\partial x},
$$

then the continuity equation (3.3) is identically satisfied. Pressure may be eliminated from the system by taking the curl of the momentum equation (3.9). This results in the vorticity equation

$$
\frac{\partial \omega}{\partial t}+\nabla \cdot(\omega \mathbf{v})=c_{p} \frac{d \pi_{0}}{d z} \frac{\partial \tilde{\theta}}{\partial x},
$$

where

$$
\omega=\hat{\mathbf{y}} \cdot \nabla \times \mathbf{v}=-\frac{1}{\rho_{0}{ }^{2}} \frac{d \rho_{0}}{d z} \frac{\partial \psi}{\partial z}+\frac{1}{\rho_{0}} \nabla^{2} \psi .
$$

The anelastic system, in two spatial dimensions, is therefore uniquely determined by (3.16) and (3.2), which may be written

$$
\begin{gathered}
\frac{\partial \omega}{\partial t}+\partial\left(\psi, \frac{\omega}{\rho_{0}}\right)=-\partial\left(\theta, c_{p} \pi_{0}\right), \\
\frac{\partial \theta}{\partial t}+\frac{1}{\rho_{0}} \partial(\psi, \theta)=0,
\end{gathered}
$$


where the Jacobian $\partial(\cdot, \cdot)$ is defined by

$$
\partial(f, g)=\frac{\partial f}{\partial z} \frac{\partial g}{\partial x}-\frac{\partial f}{\partial x} \frac{\partial g}{\partial z} .
$$

We reiterate that this representation is valid (and energetically consistent) for nonconstant $\theta_{0}(z)$.

Consider now the new decomposition of the dependent fields

$$
\begin{aligned}
\theta(x, z, t) & =\bar{\theta}(x, z)+\theta^{\prime}(x, z, t), \\
\omega(x, z, t) & =\bar{\omega}(x, z)+\omega^{\prime}(x, z, t),
\end{aligned}
$$

where the overbar represents a steady-state two-dimensional background field and primed quantities denote deviations away from this state. The disturbance fields need not be of small amplitude. (By this we mean that a low-order Taylor series expansion about the steady-state background fields may provide an inadequate representation of the disturbance.) The assumption that $(\bar{\theta}, \bar{\omega})$ is a steady solution to (3.18) implies the relations

$$
\begin{aligned}
\partial\left(\bar{\psi}, \frac{\bar{\omega}}{\rho_{0}}\right) & =-\partial\left(\bar{\theta}, c_{p} \pi_{0}\right), \\
\partial(\bar{\psi}, \bar{\theta}) & =0 .
\end{aligned}
$$

From $(3.22 b)$ it is clear that

$$
\bar{\psi}=\bar{\psi}(\bar{\theta}) \text {. }
$$

Employing this functional relation between $\bar{\theta}$ and $\bar{\psi}$, (3.22a) may be rewritten as

$$
\partial\left(\bar{\theta}, \frac{d \bar{\psi}}{d \bar{\theta}} \frac{\bar{\omega}}{\rho_{0}}+c_{p} \pi_{0}\right)=0
$$

so that

$$
\frac{d \bar{\psi}}{d \bar{\theta}} \frac{\bar{\omega}}{\rho_{0}}+c_{p} \pi_{0}=Z_{0}(\bar{\theta})
$$

for some function $Z_{0}$. This equation is quite important: it states that the particular combination of steady-state and background fields that appears on the left-hand side is a function only of the steady-state potential temperature of the system. The function $Z_{0}(\bar{\theta})$ is analogous to Long's function (Long 1953). In section 5 we shall see that $Z_{0}$ plays a crucial role in the construction of a pseudoenergy for the anelastic system.

Removing the steady-state component (3.22) from (3.18) results in the exact disturbance equations

$$
\begin{aligned}
& \omega_{t}^{\prime}=-\partial\left(\psi^{\prime}, \frac{\bar{\omega}}{\rho_{0}}\right)-\partial\left(\bar{\psi}, \frac{\omega^{\prime}}{\rho_{0}}\right) \\
&-\partial\left(\psi^{\prime}, \frac{\omega^{\prime}}{\rho_{0}}\right)-\partial\left(\theta^{\prime}, c_{p} \pi_{0}\right), \\
& \theta_{t}^{\prime}=-\frac{1}{\rho_{0}} \partial\left(\psi^{\prime}, \bar{\theta}\right)-\frac{1}{\rho_{0}} \partial\left(\bar{\psi}, \theta^{\prime}\right)-\frac{1}{\rho_{0}} \partial\left(\psi^{\prime}, \theta^{\prime}\right) .
\end{aligned}
$$

At small disturbance amplitude, the linearized equations obtain on dropping terms quadratic in the primed quantities.

\section{c. Transformation from the anelastic to the Boussinesq system}

Earlier it was stated that the Boussinesq system is a subset of the more general anelastic equations. One must take care, however, to qualify what is meant by the term "Boussinesq approximation." Benjamin $(1984,1986)$ has discussed two versions of this approximation, which one may call the "strong" and "weak" forms. The "strong" form is what is usually meant by the Boussinesq approximation within the meteorological literature. In this case one assumes

$$
\left(\frac{\partial}{\partial t}+\mathbf{v} \cdot \nabla\right) \rho=0, \quad \nabla \cdot \mathbf{v}=0
$$

hydrostatic balance of the background fields, and, except in the buoyancy term, a constant reference density in all terms of the primitive equations. The system of equations that results from such an approximation is quite obviously a subset of the more general anelastic system. In the "weak" form of the Boussinesq approximation, however, only (3.26a,b) is assumed, and the resulting system is not derivative of the anelastic equations. Therefore, in all subsequent references to the "Boussinesq" system or approximation in this paper, we shall mean the "strong" form.

In order to obtain the Boussinesq counterpart of expressions derived for the anelastic system, one must first make the straightforward substitutions

$$
\rho_{0} \rightarrow \rho_{r}, \quad \theta_{0} \rightarrow \theta_{r}, \quad \pi_{0} \rightarrow-\frac{g z}{c_{p} \theta_{r}}
$$

(where the subscript $r$ refers to some constant reference state), the last relation following directly from (3.8) in this case. For convenience we also make the replacement

$$
\psi \rightarrow \rho_{r} \psi,
$$

so that $\psi$ now becomes the conventional streamfunction. Given that the corresponding Boussinesq system is usually expressed in terms of vorticity and density rather than vorticity and potential temperature, one further elementary substitution may be performed in order to cast the results in this more familiar form. If one eliminates temperature between the ideal gas law and the definition of potential temperature, and linearizes the resulting equation, it is found that

$$
\frac{\tilde{\theta}}{\theta_{0}}=-\frac{\tilde{\rho}}{\rho_{0}}+\frac{\tilde{p}}{c^{2} \rho_{0}}
$$

where $c$ is the adiabatic sound speed. Since sound waves have been filtered from both the anelastic and Bous- 
sinesq systems, the speed of sound may be taken to be effectively infinite. Therefore, in the Boussinesq limit we may write

$$
\tilde{\theta}=-\theta_{r} \frac{\tilde{\rho}}{\rho_{r}} .
$$

The conversion from the potential temperature to density representation is thus most easily effected by the mapping

$$
\theta \rightarrow-\frac{\theta_{r}}{\rho_{r}} \rho
$$

applied to both components in the decomposition (3.20).

All of the results presented in this paper may be easily transformed into their Boussinesq counterparts by a simple application of (3.27)-(3.29).

\section{Momentum and Casimir integral invariants of the anelastic system}

In this section the Hamiltonian theory described earlier ( section 2) will be employed to derive integrals that represent total horizontal momentum and Casimir invariants specific to the anelastic system. The symmetries and associated conservation laws of the Boussinesq equations have been previously discussed by Ripa (1981) and Benjamin (1986).

\section{a. Hamiltonian structure}

In order to cast the anelastic system in the symplectic form (2.1), we take

$$
U=\left(\begin{array}{l}
\omega \\
\theta
\end{array}\right)
$$

and $\mathscr{H}$ as given by ( $3.13 \mathrm{a})$ or equivalently

$$
\mathscr{H}=\iint\left(\frac{1}{2 \rho_{0}}|\nabla \psi|^{2}+\rho_{0} c_{p} \pi_{0} \theta\right) d x d z .
$$

The functional derivatives appearing in (2.1) may be determined by considering the first variation of $\mathscr{H}$ :

$$
\begin{aligned}
\delta \mathscr{H} & =\iint\left[\frac{1}{\rho_{0}}\left(\frac{\partial \psi}{\partial x} \frac{\partial \delta \psi}{\partial x}+\frac{\partial \psi}{\partial z} \frac{\partial \delta \psi}{\partial z}\right)+\rho_{0} c_{p} \pi_{0} \delta \theta\right] d x d z \\
& =\iint\left[-\psi \delta \omega+\rho_{0} c_{p} \pi_{0} \delta \theta\right] d x d z
\end{aligned}
$$

A comparison of (4.3) with (2.2) then yields

$$
\frac{\delta \mathscr{H}}{\delta \omega}=-\psi, \quad \frac{\delta \mathscr{H}}{\delta \theta}=\rho_{0} c_{p} \pi_{0}
$$

The second equality in (4.3) is a result of an integration by parts under the assumption of simple boundary conditions (i.e., periodic boundary conditions or vanishing fields at infinity). Procedures exist by which more realistic boundary conditions may be incorporated into the problem (cf. Lewis et al. 1986; McIntyre and Shepherd 1987; Shepherd 1990, §4.1). Our primary motivation for expressing the anelastic system in Hamiltonian form, however, is merely to employ a systematic method to determine the Casimir invariants. Since a more sophisticated approach to the boundary conditions would not affect the local form of the waveactivity conservation laws (which are the principal results of this paper), such consideration has been neglected for the sake of simplicity. This issue is discussed further in section 7 .

To complete the representation of (3.18) in the Hamiltonian form (2.1), it only remains to determine the appropriate form of the symplectic matrix $J$. It may be easily verified that the substitution of

$$
J=\left(\begin{array}{cc}
\partial\left(\cdot, \frac{\omega}{\rho_{0}}\right) & \partial\left(\frac{\dot{\bar{\rho}}}{\rho_{0}}, \theta\right) \\
\frac{1}{\rho_{0}} \partial(\cdot, \theta) & 0
\end{array}\right)
$$

together with (4.1) and (4.4) into (2.1) results in the anelastic system (3.18). In order for a particular form of $J$ to be valid, however, it must be demonstrated that (2.4) $-(2.7)$ are satisfied. The first three conditions are trivial and may be seen to be satisfied by inspection. The final condition, however (Jacobi's identity), is in no way a trivial matter to verify. The fact that $J$ as given by (4.5) satisfies (2.7) is demonstrated for the reader in appendix $A$.

By applying the transformation (3.27)-(3.29) to the anelastic version of $(2.1)$, we may determine the symplectic form in the Boussinesq limit (cf. Benjamin 1986; Abarbanel et al. 1986). In this limit it is found that

$$
\frac{\delta \mathscr{H}}{\delta \omega}=-\rho_{r} \psi, \quad \frac{\delta \mathscr{H}}{\delta \rho}=g z,
$$

and

$$
J=\frac{1}{\rho_{r}}\left(\begin{array}{cc}
\partial(\cdot, \omega) & \partial(\cdot, \rho) \\
\partial(\cdot, \rho) & 0
\end{array}\right) .
$$

The expressions (4.6) and (4.7), when substituted into (2.1), result in the well-known vorticity-density representation of the Boussinesq system.

\section{b. Integral invariants}

Given the appropriate $J$ of a system, one may invoke Noether's theorem (see section 3b) to determine integral invariants associated with explicit continuous symmetries of the Hamiltonian. Through the time invariance of the Hamiltonian we have the usual result that the Hamiltonian itself represents the total energy of the system. In a similar manner, Noether's theorem may be employed to determine the form of total hor- 
izontal momentum conserved in problems where there is $x$-translational symmetry of the Hamiltonian. From (2.9) we have

$$
J \frac{\delta \mathcal{M}}{\delta U}=-U_{x}
$$

where $M$ is the total $x$ momentum in the system. A substitution of (4.1) and (4.5) into (4.8) results in the following particular solution for the functional derivatives of $\mathcal{M}$ :

$$
\frac{\delta M}{\delta \omega}=-\int \rho_{0}(z) d z, \quad \frac{\delta M}{\delta \theta}=0 .
$$

As a result we have that the total $x$ momentum in the anelastic system can be taken to be

$$
\mathcal{M}=-\iint\left[\omega \int^{z} \rho_{0}(\eta) d \eta\right] d x d z
$$

It may be noted that in (4.9) we could have let $\delta M /$ $\delta \theta=\rho_{0}$. To do so would make no difference in the end, however, since as we shall see below, the two resulting invariants differ only by a Casimir. Under the Boussinesq approximation, (4.10) reduces to

$$
\mathcal{M}=-\rho_{r} \iint z \omega d x d z .
$$

In order to derive wave-activity conservation laws from energy (4.2) or momentum (4.10), it is necessary to determine the Casimir invariants of the system. Substituting (4.1) and (4.5) into the defining relation (2.12), namely,

$$
J \frac{\delta \mathcal{C}}{\delta U}=0
$$

it is found that

$$
\frac{\delta \mathcal{C}}{\delta \omega}=f(\theta), \quad \frac{\delta \mathcal{C}}{\delta \theta}=\omega f^{\prime}(\theta)+\rho_{0} g(\theta)
$$

for arbitrary functions $f$ and $g$, the prime denoting differentiation. It follows from (4.12) that the Casimir invariants for the anelastic system can be put in the form

$$
\mathcal{e}=\iint\left[\omega C_{1}(\theta)+\rho_{0} C_{2}(\theta)\right] d x d z
$$

with $f=C_{1}$ and $g=C_{2}^{\prime}$. Similarly, employing (3.27)(3.29) we find that the Casimir invariants in the Boussinesq limit take the form

$$
\mathcal{C}=\iint\left[\omega K_{1}(\rho)+\rho_{r} K_{2}(\rho)\right] d x d z
$$

While it is not necessary to include the constant $\rho_{r}$ in the second term of the integrand, it has been retained for consistency with the anelastic system.
In the next two sections, the specific forms of $C_{1}(\theta)$ and $C_{2}(\theta)$, and of $K_{1}(\theta)$ and $K_{2}(\theta)$, are determined for the wave activities associated with pseudoenergy and pseudomomentum.

\section{Finite-amplitude pseudoenergy conservation laws}

We first consider the wave-activity conservation law (1.1) as it applies to the case of energy. In this derivation we consider the most general form of the problem, which involves flow deviations from a two-dimensional steady state.

\section{a. Specific Casimir functions}

Employing (4.2) and (4.13) in the definition of pseudoenergy (2.13), the first variation of $\mathcal{A}$ evaluated at $U=\bar{U}$ is found to be

$$
\begin{aligned}
&\left.\delta \mathcal{A}\right|_{U=\bar{U}}=\iint {\left[\left(-\bar{\psi}+C_{1}(\bar{\theta})\right) \delta \omega+\left(\rho_{0} c_{p} \pi_{0}\right.\right.} \\
&\left.\left.+\bar{\omega} C_{1}^{\prime}(\bar{\theta})+\rho_{0} C_{2}^{\prime}(\bar{\theta})\right) \delta \theta\right] d x d z,
\end{aligned}
$$

where primes on the Casimir functions denote differentiation. The specific choices for the arbitrary Casimir functions $C_{1}(\theta)$ and $C_{2}(\theta)$ that make (5.1) vanish are quite obviously

$$
\begin{gathered}
C_{1}(\bar{\theta})=\bar{\psi} \\
C_{2}^{\prime}(\bar{\theta})=-\frac{\bar{\omega}}{\rho_{0}} \frac{d \bar{\psi}}{d \bar{\theta}}-c_{p} \pi_{0}=-Z_{0}(\bar{\theta}) .
\end{gathered}
$$

The result that $\bar{\psi}$ is a function of $\bar{\theta}$, which follows from (5.2), is not a new constraint imposed by this procedure; rather, it was already implied by the steady-state relation $(3.22 b)$. Indeed, this shows that in order to construct a pseudoenergy, the basic state must be a steady solution of the dynamics. The appearance of the generalized Long's function $Z_{0}$ in the specification of $C_{2}$ is likewise no accident: while it is not at all obvious at first sight that the particular combination of quantities that appears between the two equalities in (5.3) is a function of $\bar{\theta}$ alone, it is guaranteed to be so by (3.24). We have, therefore, that the specific form of $C_{2}$ is

$$
C_{2}(\bar{\theta})=-\int Z_{0}(\bar{\theta}) d \bar{\theta} .
$$

The functions $C_{1}$ and $C_{2}$ may be viewed as functional relations between the dependent fields that comprise the steady-state flow. Since the steady-state fields are known a priori, however, we may also consider $C_{1}$ and $C_{2}$ to be just known functions of some independent variable $\eta$. In the definition (2.13) of $\mathcal{A}$ it is these specific functional forms that are employed with $\eta=\bar{\theta}$ and $\eta=\bar{\theta}+\theta^{\prime}$. 


\section{b. Finite-amplitude wave activity}

Given the specific form of the Casimir $\mathcal{C}$ we may now write down the expression for pseudoenergy. Using (5.2) and (5.4), a direct substitution of (4.2) and (4.13) into $(2.13)$ results in

$$
\begin{aligned}
\mathcal{A}=\int & \int\left\{\frac{1}{2} \rho_{0}\left|\mathbf{v}^{\prime}\right|^{2}+\rho_{0} \overline{\mathbf{v}} \cdot \mathbf{v}^{\prime}+\left(\bar{\omega}+\omega^{\prime}\right) \Psi\left(\bar{\theta}+\theta^{\prime}\right)\right. \\
& \left.-\bar{\omega} \Psi(\bar{\theta})+\rho_{0} c_{p} \pi_{0} \theta^{\prime}-\rho_{0} \int_{\bar{\theta}}^{\bar{\theta}+\theta^{\prime}} Z_{0}(\eta) d \eta\right\} d x d z,
\end{aligned}
$$

where $\Psi(\cdot)=\bar{\psi}(\cdot)$ indicates the functional dependence of $\bar{\psi}$ on $\bar{\theta}$ represented by (3.23). Even though our particular selection of Casimir functions has ensured that (5.5) is at least quadratic in the disturbance fields, it is advantageous to make this fact explicit in the expression for $\mathcal{A}$. To this end, consider the second term on the right-hand side of $(5.5)$. This term may be written

$$
\begin{aligned}
\iint \rho_{0} \overline{\mathbf{v}} \cdot \mathbf{v}^{\prime} d x d z & =\iint \frac{1}{\rho_{0}}\left(\frac{\partial \bar{\psi}}{\partial z} \frac{\partial \psi^{\prime}}{\partial z}+\frac{\partial \bar{\psi}}{\partial x} \frac{\partial \psi^{\prime}}{\partial x}\right) d x d z \\
& =\iint-\bar{\psi} \omega^{\prime} d x d z \\
& =\iint-\Psi(\bar{\theta}) \omega^{\prime} d x d z
\end{aligned}
$$

where the equality between the first and the second line is obtained through an integration by parts. Further, employing the relation (3.24) for the generalized Long's function, the fifth term in the integrand of (5.5) may be rewritten as

$$
\rho_{0} c_{p} \pi_{0} \theta^{\prime}=\rho_{0} Z_{0}(\bar{\theta}) \theta^{\prime}-\bar{\omega} \Psi^{\prime}(\bar{\theta}) \theta^{\prime},
$$

where $\Psi^{\prime}(\bar{\theta}) \equiv d \Psi / d \bar{\theta}$. Substituting (5.6) and (5.7) into (5.5) we find that the integrand, or the local density $A$ of the wave activity, becomes

$$
\begin{aligned}
A=\frac{1}{2 \rho_{0}} \mid \nabla & \left.\psi^{\prime}\right|^{2}+\left(\bar{\omega}+\omega^{\prime}\right)\left(\Psi\left(\bar{\theta}+\theta^{\prime}\right)\right. \\
- & \left.\Psi(\bar{\theta})-\Psi^{\prime}(\bar{\theta}) \theta^{\prime}\right)+\Psi^{\prime}(\bar{\theta}) \omega^{\prime} \theta^{\prime} \\
& -\rho_{0} \int_{0}^{\theta^{\prime}}\left[Z_{0}(\bar{\theta}+\eta)-Z_{0}(\bar{\theta})\right] d \eta,
\end{aligned}
$$

which is explicitly of quadratic order in disturbance quantities.
In the special case where the steady basic flow is chosen to be the resting background state $\pi_{0}, \theta_{0}$, we have $\Psi \equiv 0, \bar{\omega} \equiv 0$, and $Z_{0}(\bar{\theta})=c_{p} \pi_{0}\left(\theta_{0}\right)$. It follows immediately that $(5.8)$ reduces to $(3.13 \mathrm{~b})$, verifying that the latter is indeed the pseudoenergy relative to the (resting) background state.

\section{c. Small-amplitude local wave-activity conservation law}

Following the procedure outlined in section $2 \mathrm{c}$, we now wish to express the time derivative of the local wave-activity density $A$ in the flux form

$$
\frac{\partial A}{\partial t}=-\nabla \cdot \mathbf{F} .
$$

In order to remove some of the arbitrariness in this expression, we will impose the additional requirement that, in the WKB limit, (5.9) reduce to the condition (2.16). Toward this end we first consider the smallamplitude counterparts of $A$ and $\mathbf{F}$ and determine their appropriate forms such that $(2.16)$ is satisfied in the relevant limit. We will then be in a position to derive finite-amplitude expressions for these quantities that are consistent with these small-amplitude expressions and therefore with (2.16).

At small disturbance amplitude, an expression for $A$ may be determined in two manners. In the first, we simply consider the lowest-order contribution to (5.8) after performing a Taylor series expansion of $\Psi\left(\bar{\theta}+\theta^{\prime}\right)$ and $Z_{0}(\bar{\theta}+\eta)$ about $\theta=\bar{\theta}$. The small-amplitude expression for $A$ is then determined to be

$$
\begin{aligned}
A=\frac{1}{2 \rho_{0}}\left|\nabla \psi^{\prime}\right|^{2}+\left(\frac{\bar{\omega}}{2} \Psi^{\prime \prime}(\bar{\theta})-\right. & \left.\frac{\rho_{0}}{2} Z_{0}^{\prime}(\bar{\theta})\right)\left(\theta^{\prime}\right)^{2} \\
& +\Psi^{\prime}(\bar{\theta}) \omega^{\prime} \theta^{\prime}
\end{aligned}
$$

A second way to realize this expression would be to consider the expansion of $\mathcal{A}$ :

$$
\mathcal{A}=\left.\mathcal{A}\right|_{U=\bar{U}}+\left.\delta \mathcal{A}\right|_{U=\bar{U}}+\left.\frac{1}{2} \delta^{2} \mathcal{A}\right|_{U=\bar{U}}+\cdots
$$

By construction we have that the first two terms on the right-hand side of $(5.11)$ are zero. Therefore, the leading contribution to $\mathcal{A}$ may be obtained by calculating its second variation. Performing this calculation we have:

$$
\begin{aligned}
\left.\frac{1}{2} \delta^{2} \mathcal{A}\right|_{U=\bar{U}} & =\left.\frac{1}{2} \delta\left\{\iint\left[C_{1}(\theta) \delta \omega+\frac{1}{\rho_{0}} \nabla \psi \cdot \nabla \delta \psi+\left(\rho_{0} c_{p} \pi_{0}+\omega C_{1}^{\prime}(\theta)+\rho_{0} C_{2}^{\prime}(\theta)\right) \delta \theta\right] d x d z\right\}\right|_{U=\bar{U}} \\
& =\iint\left\{\frac{1}{2 \rho_{0}}|\nabla \delta \psi|^{2}+C_{1}^{\prime}(\bar{\theta}) \delta \omega \delta \theta+\frac{1}{2}\left(\rho_{0} C_{2}^{\prime \prime}(\bar{\theta})+\bar{\omega} C_{1}^{\prime \prime}(\bar{\theta})\right)(\delta \theta)^{2}\right\} d x d z .
\end{aligned}
$$


A simple substitution of (5.2) and (5.4) into the above expression reveals that the integrand is equivalent to (5.10). The advantage of this second method is that we need not explicitly consider the finite-amplitude expression for $A$ in order to derive its small-amplitude counterpart.

Constructing now the small-amplitude form of (1.1), from $(5.10)$ we have

$$
\begin{aligned}
\frac{\partial A}{\partial t}=\left(-\psi^{\prime}\right. & \left.+\Psi^{\prime}(\bar{\theta}) \theta^{\prime}\right) \omega_{t}^{\prime}+\left(\Psi^{\prime}(\bar{\theta}) \omega^{\prime}+\left\{\bar{\omega} \Psi^{\prime \prime}(\bar{\theta})\right.\right. \\
& \left.\left.-\rho_{0} Z_{0}^{\prime}(\bar{\theta})\right\} \theta^{\prime}\right) \theta_{t}^{\prime}+\nabla \cdot\left(\frac{\psi^{\prime}}{\rho_{0}} \nabla \psi_{t}^{\prime}\right)
\end{aligned}
$$

In deriving this expression we have used the result that

$$
\frac{\partial}{\partial t}\left(\frac{1}{2 \rho_{0}}\left|\nabla \psi^{\prime}\right|^{2}\right)=\nabla \cdot\left(\frac{\psi^{\prime}}{\rho_{0}} \nabla \psi_{t}^{\prime}\right)-\psi^{\prime} \omega_{t}^{\prime}
$$

In this form, we need not explicitly deal with the local time derivative of $\psi^{\prime}$ since the term that contains this quantity already appears in flux form in (5.13). However, as a consequence the flux $\mathbf{F}$ will contain the explicit time derivative of $\psi^{\prime}$, making it a more difficult quantity to calculate. This parallels the situation for barotropic and quasigeostrophic baroclinic flow (McIntyre and Shepherd 1987).

The local time derivatives of $\omega^{\prime}$ and $\theta^{\prime}$ may be eliminated from the right-hand side of $(5.13)$ by substitution from the linearized form of the anelastic disturbance equations (3.25). These are, respectively,

$$
\begin{array}{r}
\omega_{t}^{\prime}=-\partial\left(\psi^{\prime}, \frac{\bar{\omega}}{\rho_{0}}\right)-\partial\left(\bar{\psi}, \frac{\omega^{\prime}}{\rho_{0}}\right)-\partial\left(\theta^{\prime}, c_{p} \pi_{0}\right), \\
\theta_{t}^{\prime}=-\frac{1}{\rho_{0}} \partial\left(\psi^{\prime}, \bar{\theta}\right)-\frac{1}{\rho_{0}} \partial\left(\bar{\psi}, \theta^{\prime}\right) .
\end{array}
$$

Upon substitution of (5.15), the right-hand side of (5.13) may be written (after some manipulation) in the form

$$
\begin{aligned}
\frac{\partial A}{\partial t}= & -\frac{1}{2} \partial\left(\frac{\bar{\omega}}{\rho_{0}},\left(\psi^{\prime}\right)^{2}\right)+\partial\left(\bar{\psi}, \frac{\psi^{\prime} \omega^{\prime}}{\rho_{0}}\right) \\
& -\partial\left(c_{p} \pi_{0}, \theta^{\prime} \psi^{\prime}\right)-\partial\left(\bar{\theta}, \frac{\omega^{\prime} \theta^{\prime}}{\rho_{0}}\left(\Psi^{\prime}(\bar{\theta})\right)^{2}\right) \\
& +\partial\left(\Psi^{\prime}(\bar{\theta}) \frac{\bar{\omega}}{\rho_{0}}, \Psi^{\prime}(\bar{\theta})\left(\theta^{\prime}\right)^{2}\right) \\
& +\partial\left(c_{p} \pi_{0}, \Psi^{\prime}(\bar{\theta})\left(\theta^{\prime}\right)^{2}\right) \\
& -\frac{1}{2} \partial\left(\frac{\bar{\omega}}{\rho_{0}}\left(\Psi^{\prime}(\bar{\theta})\right)^{2},\left(\theta^{\prime}\right)^{2}\right) \\
& +\nabla \cdot\left(\frac{\psi^{\prime}}{\rho_{0}} \nabla \psi_{t}^{\prime}\right)=-\nabla \cdot \mathbf{F} .
\end{aligned}
$$

The arbitrary nature of the pseudoenergy flux $\mathbf{F}$ is clearly evident in (5.16). The flux form of any Jacobian may be written as

$$
\begin{aligned}
& \partial(f, g)=\partial_{z}\left(f \partial_{x} g\right)-\partial_{x}\left(f \partial_{z} g\right), \\
& \partial(f, g)=-\partial_{z}\left(g \partial_{x} f\right)+\partial_{x}\left(g \partial_{z} f\right),
\end{aligned}
$$

or any normalized linear combination of these two forms. This problem also arises when one attempts to determine the form of the finite-amplitude flux from the full $A$. It is for this reason that it is best to first work in the small-amplitude limit to determine the most appropriate decomposition of $A_{t}$ into $-\nabla \cdot \mathbf{F}$.

In order to satisfy (2.16), each Jacobian in (5.16) is decomposed into flux form via (5.17b), and, in addition, the arbitrary nature of the local wave-activity conservation law (1.1) is exploited as discussed in section $2 \mathrm{c}$. In particular, we append to the wave activity the divergence of the vector

$$
\mathbf{B}=\left(\frac{-\psi^{\prime}}{2 \rho_{0}} \frac{\partial \psi^{\prime}}{\partial x}, \frac{-\psi^{\prime}}{2 \rho_{0}} \frac{\partial \psi^{\prime}}{\partial z}\right)
$$

while subtracting its time derivative $\mathbf{B}_{t}$ from the flux. In doing so, the conservation law (1.1) is left unaltered and the small-amplitude relation (2.16) is then satisfied. This is demonstrated for the reader in appen$\operatorname{dix} B$.

When the basic flow is nonparallel, (2.16) is only valid for disturbances with vertical wavelengths $\lambda_{2}$ restricted according to

$$
\lambda_{z} \ll \frac{4 \pi}{3}\left(\frac{1}{\rho_{0}} \frac{d \rho_{0}}{d z}\right)^{-1} .
$$

For an isothermal atmosphere, this constraint reduces to $\lambda_{z} \ll 4 H$ where $H$ is the density scale height. In practice, we may expect disturbances with wavelengths of up to one scale height to still satisfy this restriction. When the basic flow is parallel, on the other hand, the condition (5.19) is not required.

As a result of imposing the constraint (2.16), therefore, it is clear that, in addition to modifying the flux $\mathbf{F}$, one must also alter the expressions for wave activity derived in both the finite-amplitude (5.8) and smallamplitude (5.10) regimes. The addition of $\nabla \cdot \mathbf{B}$ to (5.8) results in the form for the finite-amplitude wave activity

$$
\begin{array}{r}
A=\frac{-\psi^{\prime} \omega^{\prime}}{2}+\left(\bar{\omega}+\omega^{\prime}\right)\left(\Psi\left(\bar{\theta}+\theta^{\prime}\right)-\Psi(\bar{\theta})-\Psi^{\prime}(\bar{\theta}) \theta^{\prime}\right) \\
+\Psi^{\prime}(\bar{\theta}) \omega^{\prime} \theta^{\prime}-\rho_{0} \int_{0}^{\theta^{\prime}}\left[Z_{0}(\bar{\theta}+\eta)-Z_{0}(\bar{\theta})\right] d \eta
\end{array}
$$


while its addition to (5.10) provides the appropriate small-amplitude representation

$$
\begin{array}{r}
A=\frac{-\psi^{\prime} \omega^{\prime}}{2}+\left(\frac{\bar{\omega}}{2} \Psi^{\prime \prime}(\bar{\theta})-\frac{\rho_{0}}{2} Z_{0}^{\prime}(\bar{\theta})\right)\left(\theta^{\prime}\right)^{2} \\
+\Psi^{\prime}(\bar{\theta}) \omega^{\prime} \theta^{\prime} .
\end{array}
$$

In deriving these expressions we have made use of the fact that

$$
\frac{-\psi^{\prime} \omega^{\prime}}{2}=\frac{1}{2 \rho_{0}}\left|\nabla \psi^{\prime}\right|^{2}+\nabla \cdot \mathbf{B} .
$$

It is important to point out that one may obtain (5.20) through less elaborate means. A simple integration by parts, of the type performed in (5.6), on the first term in (5.5) would result in forms identical to (5.20) without the explicit introduction of the device $\mathbf{B}$.

In deriving the analogous results under the Boussinesq approximation, it turns out that there is no need to modify $A$ in any special manner in order to satisfy (2.16); this is due to the fact that when averaging over phase in the WKB limit, the quantity $\nabla \cdot B$ vanishes. Hence, we may simply use the transformation (3.27)(3.29) on (5.8) to obtain the Boussinesq representation of the finite-amplitude pseudoenergy,

$$
\begin{aligned}
A= & \rho_{r}\left\{\frac{1}{2}\left|\nabla \psi^{\prime}\right|^{2}+\left(\bar{\omega}+\omega^{\prime}\right)\left(\Psi\left(\bar{\rho}+\rho^{\prime}\right)\right.\right. \\
& \left.-\Psi(\bar{\rho})-\Psi^{\prime}(\bar{\rho}) \rho^{\prime}\right)+\Psi^{\prime}(\bar{\rho}) \omega^{\prime} \rho^{\prime} \\
& \left.\quad-\int_{0}^{\rho^{\prime}}\left[Z_{0}(\bar{\rho}+\tilde{\rho})-Z_{0}(\bar{\rho})\right] d \tilde{\rho}\right\} .
\end{aligned}
$$

In deriving (5.21a), we have used the relation

$$
Z_{0}(\bar{\theta}) \rightarrow-\frac{\rho_{r}}{\theta_{r}} Z_{0}(\bar{\rho})=-\frac{\rho_{r}}{\theta_{r}}\left(\Psi^{\prime}(\bar{\rho}) \bar{\omega}+\frac{g z}{\rho_{r}}\right),
$$

which is obtained through the transformation of (3.24). The corresponding small-amplitude local wave-activity density under the Boussinesq approximation is then

$$
\begin{array}{r}
A=\rho_{r}\left[\frac{1}{2}\left|\nabla \psi^{\prime}\right|^{2}+\left(\frac{\bar{\omega}}{2} \Psi^{\prime \prime}(\bar{\rho})-\frac{1}{2} Z_{0}^{\prime}(\bar{\rho})\right)\left(\rho^{\prime}\right)^{2}\right. \\
\left.+\Psi^{\prime}(\bar{\rho}) \omega^{\prime} \rho^{\prime}\right]
\end{array}
$$

This expression corresponds to those given in equations (2.28) of Abarbanel et al. (1986) and (1.9) of Vladimirov (1987).
In appendix $B$ it is demonstrated that the appropriate form of the pseudoenergy flux at small amplitude in the anelastic representation is

$$
\begin{aligned}
F_{(x)}= & \frac{\left(\psi^{\prime}\right)^{2}}{2} \partial_{z}\left(\frac{\bar{\omega}}{\rho_{0}}\right)-\bar{u} \psi^{\prime} \omega^{\prime}-\frac{g}{\theta_{0}} \theta^{\prime} \psi^{\prime}+\bar{u} \Psi^{\prime}(\bar{\theta}) \omega^{\prime} \theta^{\prime} \\
& +\frac{1}{2}\left(\theta^{\prime}\right)^{2}\left(\frac{g}{\theta_{0}} \Psi^{\prime}(\bar{\theta})+\bar{u} \bar{\omega} \Psi^{\prime \prime}(\bar{\theta})-\rho_{0} \bar{u} Z_{0}^{\prime}(\bar{\theta})\right) \\
& -\frac{\psi^{\prime}}{2 \rho_{0}} \partial_{x} \psi_{t}^{\prime}+\frac{\psi_{t}^{\prime}}{2 \rho_{0}} \partial_{x} \psi^{\prime}, \quad(5.22 \mathrm{a} \\
F_{(z)}= & -\frac{\left(\psi^{\prime}\right)^{2}}{2} \partial_{x}\left(\frac{\bar{\omega}}{\rho_{0}}\right)-\bar{w} \psi^{\prime} \omega^{\prime}+\bar{w} \Psi^{\prime}(\bar{\theta}) \omega^{\prime} \theta^{\prime} \\
+ & \frac{1}{2}\left(\theta^{\prime}\right)^{2}\left(\bar{w} \bar{\omega} \Psi^{\prime \prime}(\bar{\theta})-\rho_{0} \bar{w} Z_{0}^{\prime}(\bar{\theta})\right) \\
& -\frac{\psi^{\prime}}{2 \rho_{0}} \partial_{z} \psi_{t}^{\prime}+\frac{\psi_{t}^{\prime}}{2 \rho_{0}} \partial_{z} \psi^{\prime} .
\end{aligned}
$$

Under the Boussinesq approximation we have the corresponding result

$$
\begin{aligned}
F_{(x)}= & \rho_{r}\left[\frac{\left(\psi^{\prime}\right)^{2}}{2} \partial_{z} \bar{\omega}-\bar{u} \psi^{\prime} \omega^{\prime}+g \frac{\rho^{\prime}}{\rho_{r}} \psi^{\prime}\right. \\
+ & \bar{u} \Psi^{\prime}(\bar{\rho}) \omega^{\prime} \rho^{\prime}+\frac{1}{2}\left(\rho^{\prime}\right)^{2}\left(-\frac{g}{\rho_{r}} \Psi^{\prime}(\bar{\rho})\right. \\
& \left.\left.+\bar{u} \bar{\omega} \Psi^{\prime \prime}(\bar{\rho})-\bar{u} Z_{0}^{\prime}(\bar{\rho})\right)-\psi^{\prime} \partial_{x} \psi_{t}^{\prime}\right], \quad \\
F_{(z)}= & \rho_{r}\left[-\frac{\left(\psi^{\prime}\right)^{2}}{2} \partial_{x} \bar{\omega}-\bar{w} \psi^{\prime} \omega^{\prime}+\bar{w} \Psi^{\prime}(\bar{\rho}) \omega^{\prime} \rho^{\prime}\right. \\
+ & \left.\frac{1}{2}\left(\rho^{\prime}\right)^{2}\left(\bar{w} \bar{\omega} \Psi^{\prime \prime}(\bar{\rho})-\bar{w} Z_{0}^{\prime}(\bar{\rho})\right)-\psi^{\prime} \partial_{z} \psi_{t}^{\prime}\right] .
\end{aligned}
$$

\section{d. Finite-amplitude local conservation law}

In this section the finite-amplitude counterparts of (5.22) and (5.23) are determined. In this endeavor it is advantageous to perform the trichotomy

$$
A=A_{1}+A_{2}+\nabla \cdot \mathbf{B}
$$

of $(5.20 \mathrm{a})$, where

$$
\begin{gathered}
A_{1}=\frac{1}{2 \rho_{0}}\left|\nabla \psi^{\prime}\right|^{2} \\
A_{2}=\left(\bar{\omega}+\omega^{\prime}\right)\left(\Psi\left(\bar{\theta}+\theta^{\prime}\right)-\Psi(\bar{\theta})-\Psi^{\prime}(\bar{\theta}) \theta^{\prime}\right) \\
+\Psi^{\prime}(\bar{\theta}) \omega^{\prime} \theta^{\prime}-\rho_{0} \int_{0}^{\theta^{\prime}}\left[Z_{0}(\bar{\theta}+\eta)-Z_{0}(\bar{\theta})\right] d \eta
\end{gathered}
$$


and $\mathrm{B}$ is given by (5.18). Considering $A_{1}$ first, we may write using (5.14)

$$
\begin{aligned}
\frac{\partial A_{1}}{\partial t}=\nabla \cdot\left(\frac{\psi^{\prime}}{\rho_{0}} \nabla \psi_{t}^{\prime}\right)-\psi^{\prime} \omega_{t}^{\prime} \\
=\nabla \cdot\left(\frac{\psi^{\prime}}{\rho_{0}} \nabla \psi_{t}^{\prime}\right)+\partial\left(\frac{\left(\psi^{\prime}\right)^{2}}{2}, \frac{\bar{\omega}+\omega^{\prime}}{\rho_{0}}\right) \\
+\psi^{\prime} \partial\left(\bar{\psi}, \frac{\omega^{\prime}}{\rho_{0}}\right)+\psi^{\prime} \partial\left(\theta^{\prime}, c_{p} \pi_{0}\right) .
\end{aligned}
$$

In (5.26) the second equality results from employing the disturbance equation (3.25a). Terms appear in (5.26) that do not easily transform into flux formnamely, the last two. Any manipulation of this expression will always result in some such terms. We thus seek identical terms of opposite sign in the corresponding expression for $\partial A_{2} / \partial t$ so that these terms will cancel when the total $\partial A / \partial t$ is constructed.

The calculation of the local time derivative of $A_{2}$ is rather involved and is provided for the reader in appendix $\mathrm{C}$. The result of this calculation is

$$
\begin{aligned}
\frac{\partial A_{2}}{\partial t}= & -\partial\left(\bar{\psi}+\psi^{\prime}, \frac{A_{2}}{\rho_{0}}\right) \\
& +\frac{\partial}{\partial x}\left\{-\frac{g}{\theta_{0}} \int_{0}^{\theta^{\prime}}[\Psi(\bar{\theta}+\eta)-\Psi(\bar{\theta})] d \eta\right\} \\
& -\partial\left(c_{p} \pi_{0}, \psi^{\prime} \theta^{\prime}\right)+\partial\left(\bar{\psi}, \frac{\omega^{\prime} \psi^{\prime}}{\rho_{0}}\right)-\psi^{\prime} \partial\left(\bar{\psi}, \frac{\omega^{\prime}}{\rho_{0}}\right) \\
& -\psi^{\prime} \partial\left(\theta^{\prime}, c_{p} \pi_{0}\right) .
\end{aligned}
$$

Adding (5.26) and (5.27) yields

$$
\begin{aligned}
& \frac{\partial A}{\partial t}=-\partial\left(\bar{\psi}+\psi^{\prime}, \frac{A_{2}}{\rho_{0}}\right) \\
&+\frac{\partial}{\partial x}\left\{-\frac{g}{\theta_{0}} \int_{0}^{\theta^{\prime}}[\Psi(\bar{\theta}+\eta)-\Psi(\bar{\theta})] d \eta\right\} \\
&-\partial\left(c_{p} \pi_{0}, \psi^{\prime} \theta^{\prime}\right)+\partial\left(\bar{\psi}, \frac{\omega^{\prime} \psi^{\prime}}{\rho_{0}}\right) \\
&-\partial\left(\frac{\bar{\omega}+\omega^{\prime}}{\rho_{0}}, \frac{\left(\psi^{\prime}\right)^{2}}{2}\right)+\nabla \cdot\left(\frac{\psi^{\prime}}{\rho_{0}} \nabla \psi_{t}^{\prime}\right)+\nabla \cdot \mathbf{B}_{t} \\
&=-\nabla \cdot \mathbf{F} .
\end{aligned}
$$

As in the small-amplitude case, the arbitrary nature of the flux is clearly evident in the expression above. Given the manner in which each Jacobian has been written in (5.28), a decomposition into flux form via (5.17b) results in a flux that, at small amplitude, is identical to (5.22) and is therefore consistent with (2.16). The finite-amplitude pseudoenergy flux is then

$$
\begin{gathered}
F_{(x)=}\left(\bar{u}+u^{\prime}\right) A_{2}+\frac{g}{\theta_{0}} \int_{0}^{\theta^{\prime}}[\Psi(\bar{\theta}+\eta)-\Psi(\bar{\theta})] d \eta \\
-\frac{g}{\theta_{0}} \psi^{\prime} \theta^{\prime}-\bar{u} \psi^{\prime} \omega^{\prime}+\partial_{z}\left(\frac{\bar{\omega}+\omega^{\prime}}{\rho_{0}}\right) \frac{\left(\psi^{\prime}\right)^{2}}{2} \\
-\frac{\psi^{\prime}}{2 \rho_{0}} \partial_{x} \psi_{t}^{\prime}+\frac{\psi_{t}^{\prime}}{2 \rho_{0}} \partial_{x} \psi^{\prime}, \quad \\
F_{(z)}=\left(\bar{w}+w^{\prime}\right) A_{2}-\bar{w} \psi^{\prime} \omega^{\prime}-\partial_{x}\left(\frac{\bar{\omega}+\omega^{\prime}}{\rho_{0}}\right) \frac{\left(\psi^{\prime}\right)^{2}}{2} \\
-\frac{\psi^{\prime}}{2 \rho_{0}} \partial_{z} \psi_{t}^{\prime}+\frac{\psi_{t}^{\prime}}{2 \rho_{0}} \partial_{z} \psi^{\prime}, \quad
\end{gathered}
$$

with $A_{2}$ given by (5.25). A similar calculation under the Boussinesq approximation leads to

$$
\begin{gathered}
F_{(x)}=\left(\bar{u}+u^{\prime}\right) A_{2}-g \int_{0}^{\rho^{\prime}}[\Psi(\bar{\rho}+\tilde{\rho})-\Psi(\bar{\rho})] d \tilde{\rho} \\
+g \psi^{\prime} \rho^{\prime}-\rho_{r} \bar{u} \psi^{\prime} \omega^{\prime}+\rho_{r} \partial_{z}\left(\bar{\omega}+\omega^{\prime}\right) \frac{\left(\psi^{\prime}\right)^{2}}{2}-\rho_{r} \psi^{\prime} \partial_{x} \psi_{t}^{\prime}, \\
F_{(z)}=\left(\bar{w}+w^{\prime}\right) A_{2}-\rho_{r} \bar{w} \psi^{\prime} \omega^{\prime} \\
\quad-\rho_{r} \partial_{x}\left(\bar{\omega}+\omega^{\prime}\right) \frac{\left(\psi^{\prime}\right)^{2}}{2}-\rho_{r} \psi^{\prime} \partial_{z} \psi_{t}^{\prime},
\end{gathered}
$$

where $A_{2}$ is the Boussinesq counterpart to (5.25).

\section{Finite-amplitude pseudomomentum conservation laws}

In this section we derive finite-amplitude wave-activity conservation laws of the form (1.1) based on the horizontal momentum. Implicit in this analysis is the assumption that the disturbance Hamiltonian is invariant under translations in the $x$ direction. As a result, the most general steady-state configuration that may be considered is the case of parallel flow, for which the steady-state fields $\bar{\psi}, \bar{\omega}$, and $\bar{\theta}$ are functions of the vertical coordinate $z$ alone.

\section{a. Specific Casimir functions}

In the definition of wave activity (2.13) we now substitute $\mathcal{M}$ for $\mathscr{H}$. Again, we require the first variation of this quantity $\delta \mathcal{A}$ to vanish so that the pseudomomentum will be at least quadratic in the disturbance fields. Imposing this condition, from (4.10) and (4.13) we have

$$
\begin{aligned}
\left.\delta \mathcal{A}\right|_{U=\bar{U}} & =\iint\left[\left\{-\int^{z} \rho_{0}(\eta) d \eta+C_{1}(\bar{\theta})\right\} \delta \omega\right. \\
& \left.+\left\{\bar{\omega} C_{1}^{\prime}(\bar{\theta})+\rho_{0} C_{2}^{\prime}(\bar{\theta})\right\} \delta \theta\right] d x d z=0 .
\end{aligned}
$$


The specific choices of the Casimir functions that satisfy (6.1) are

$$
\begin{aligned}
C_{1}(\bar{\theta}) & =\int \rho_{0}(z) d z \\
& =\int R(\bar{\theta}) d \bar{\theta}
\end{aligned}
$$

where

$$
R(\bar{\theta})=\frac{g \rho_{0}}{\theta_{0} N^{2}},
$$

and

$$
C_{2}^{\prime}(\bar{\theta})=-\frac{\bar{\omega}}{\rho_{0}} \frac{d}{d \bar{\theta}} \int \rho_{0}(z) d z=-\frac{g \bar{\omega}}{\theta_{0} N^{2}} .
$$

In deriving (6.3) and (6.5) we have used the definition

$$
N^{2} \equiv \frac{g}{\theta_{0}} \frac{d \bar{\theta}}{d z},
$$

which is the square of the steady-state Brunt-Väisälä frequency. The Casimir $C_{2}(\bar{\theta})$ is then

$$
C_{2}(\bar{\theta})=-\int S(\bar{\theta}) d \bar{\theta}
$$

where

$$
S(\bar{\theta})=\frac{g \bar{\omega}}{\theta_{0} N^{2}} .
$$

The right-hand sides of (6.4) and (6.7) are functions of $z$, and therefore may be considered functions of $\bar{\theta}$ since $z=z(\vec{\theta})$ for parallel flow.

\section{b. Finite-amplitude wave activity}

A direct substitution of (6.3) and (6.6) into the definition of pseudomomentum results in the form

$$
\begin{aligned}
\mathcal{A}=\iint\left\{\left(\bar{\omega}+\omega^{\prime}\right)\right. & \int_{0}^{\theta^{\prime}} R(\bar{\theta}+\eta) d \eta \\
& \left.-\rho_{0} \int_{0}^{\theta^{\prime}} S(\bar{\theta}+\eta) d \eta\right\} d x d z .
\end{aligned}
$$

If we note that

$$
\bar{\omega} \int_{0}^{\theta^{\prime}} R(\bar{\theta}) d \eta=\frac{\bar{\omega} g \rho_{0}}{\theta_{0} N^{2}} \theta^{\prime}=\rho_{0} \int_{0}^{\theta^{\prime}} S(\bar{\theta}) d \eta,
$$

then the integrand of (6.8) may be written in the explicitly quadratic form

$$
\begin{aligned}
& A=\left(\bar{\omega}+\omega^{\prime}\right) \int_{0}^{\theta^{\prime}}[R(\bar{\theta}+\eta)-R(\bar{\theta})] d \eta \\
& -\rho_{0} \int_{0}^{\theta^{\prime}}[S(\bar{\theta}+\eta)-S(\bar{\theta})] d \eta+R(\bar{\theta}) \theta^{\prime} \omega^{\prime} .
\end{aligned}
$$

In deriving the Boussinesq counterpart of $(6.10)$ we must be careful to employ the correct forms of the specific Casimir functions. These are:

$$
K_{1}(\bar{\rho})=\rho_{r} z \Rightarrow z=Z(\bar{\rho}),
$$

and

$$
K_{2}^{\prime}(\bar{\rho})=-\bar{\omega} \frac{d Z(\bar{\rho})}{d \bar{\rho}} \Longrightarrow K_{2}(\bar{\rho})=-\int \chi(\bar{\rho}) d \bar{\rho},
$$

where

$$
\chi(\bar{\rho})=\bar{\omega} \frac{d Z(\bar{\rho})}{d \bar{\rho}} .
$$

We have then that the local density of the finite-amplitude pseudomomentum in the Boussinesq approximation takes the form

$$
\begin{aligned}
A & =\rho_{r}\left(\bar{\omega}+\omega^{\prime}\right)\left[Z\left(\bar{\rho}+\rho^{\prime}\right)-\mathcal{Z}(\bar{\rho})-\frac{d Z(\bar{\rho})}{d \bar{\rho}} \rho^{\prime}\right] \\
& -\rho_{r} \int_{0}^{\rho^{\prime}}[\chi(\bar{\rho}+\tilde{\rho})-\chi(\bar{\rho})] d \tilde{\rho}+\rho_{r} \frac{d Z(\bar{\rho})}{d \bar{\rho}} \rho^{\prime} \omega^{\prime} .
\end{aligned}
$$

Apart from a factor of $\rho_{r}$, this corresponds to the expression (5.13) of Shepherd (1990).

\section{c. Small-amplitude local wave-activity conservation law}

As in section $5 \mathrm{c}$ we may derive the small-amplitude form of the wave activity by two methods. A Taylor series expansion of $R(\bar{\theta}+\eta)$ and $S(\bar{\theta}+\eta)$ may be substituted into (6.10) and terms of up to second order retained, or one-half of the second variation of $\mathcal{A}$ may be evaluated. The result of either of these calculations yields the small-amplitude approximation to the wave activity

$$
A=\frac{g \rho_{0}}{\theta_{0} N^{2}} \theta^{\prime} \omega^{\prime}-\frac{g^{2} \rho_{0}^{2}}{2 \theta_{0}^{2} N^{4}} \frac{d}{d z}\left(\frac{\bar{\omega}}{\rho_{0}}\right)\left(\theta^{\prime}\right)^{2} .
$$

The corresponding form under the Boussinesq approximation is

$$
A=-\frac{g}{N^{2}} \rho^{\prime} \omega^{\prime}-\frac{g^{2}}{2 \rho_{r} N^{4}} \frac{d \bar{\omega}}{d z}\left(\rho^{\prime}\right)^{2} .
$$

If we now take the local time derivative of (6.15) and eliminate local time derivatives of $\omega^{\prime}$ and $\theta^{\prime}$ by the linearized form of the disturbance equations (3.25), we find

$$
\begin{aligned}
\frac{\partial A}{\partial t} & =-\frac{\partial}{\partial x}\left\{\frac{g \rho_{0} \bar{u}}{\theta_{0} N^{2}} \theta^{\prime} \omega^{\prime}\right\} \\
& +\frac{\partial}{\partial x}\left\{\frac{g^{2} \rho_{0}}{2 \theta_{0}^{2} N^{2}}\left[-1+\frac{\rho_{0} \bar{u}}{N^{2}} \frac{d}{d z}\left(\frac{\bar{\omega}}{\rho_{0}}\right)\right]\left(\theta^{\prime}\right)^{2}\right\}+\omega^{\prime} \frac{\partial \psi^{\prime}}{\partial x} .
\end{aligned}
$$


This expression is almost in flux form already. All that remains is the $\omega^{\prime} \partial_{x} \psi^{\prime}$ term. Now, the most natural way to write this final term in flux form is

$$
\begin{aligned}
& \omega^{\prime} \frac{\partial \psi^{\prime}}{\partial x}=\frac{\partial}{\partial z}\left\{\frac{1}{\rho_{0}} \frac{\partial \psi^{\prime}}{\partial z} \frac{\partial \psi^{\prime}}{\partial x}\right\} \\
&+\frac{\partial}{\partial x}\left\{\frac{1}{2 \rho_{0}}\left[\left(\frac{\partial \psi^{\prime}}{\partial x}\right)^{2}-\left(\frac{\partial \psi^{\prime}}{\partial z}\right)^{2}\right]\right\} ;
\end{aligned}
$$

this is, for example, the analog to the form used in the barotropic case [Killworth and McIntyre 1985, Eq. (5.17)]. A decomposition of the $\omega^{\prime} \partial_{x} \psi^{\prime}$ term in this manner does not, however, satisfy the group-velocity relation (2.16). In order for the flux to be consistent with this condition in the WKB limit, this term must instead be written as

$$
\begin{aligned}
\omega^{\prime} \frac{\partial \psi^{\prime}}{\partial x}= & \frac{\partial}{\partial z}\left\{\left(\frac{\partial}{\partial z} \frac{\psi^{\prime}}{\rho_{0}{ }^{1 / 2}}\right)\left(\frac{\partial}{\partial x} \frac{\psi^{\prime}}{\rho_{0}{ }^{1 / 2}}\right)\right\} \\
& +\frac{1}{2} \frac{\partial}{\partial x}\left\{\left(\frac{\partial}{\partial x} \frac{\psi^{\prime}}{\rho_{0}{ }^{1 / 2}}\right)^{2}-\left(\frac{\partial}{\partial z} \frac{\psi^{\prime}}{\rho_{0}{ }^{1 / 2}}\right)^{2}\right. \\
& \left.+\left[\frac{1}{2 \rho_{0}} \frac{d^{2} \rho_{0}}{d z^{2}}-\frac{3}{4 \rho_{0}^{2}}\left(\frac{d \rho_{0}}{d z}\right)^{2}\right] \frac{\left(\psi^{\prime}\right)^{2}}{\rho_{0}}\right\} .
\end{aligned}
$$

The only difference between these two expressions is that in (6.18) a quantity with zero divergence has been appended to the flux. Without the constraint imposed by (2.16) we would have no reason to suspect that this quantity was a necessary component of the flux. Therefore, at small amplitude we have that the pseudomomentum flux is

$$
\begin{gathered}
F_{(x)}=\frac{g \rho_{0} \bar{u}}{\theta_{0} N^{2}} \theta^{\prime} \omega^{\prime}+\frac{g^{2} \rho_{0}}{2 \theta_{0}^{2} N^{2}}\left[1-\frac{\rho_{0} \bar{u}}{N^{2}} \frac{d}{d z}\left(\frac{\bar{\omega}}{\rho_{0}}\right)\right]\left(\theta^{\prime}\right)^{2} \\
-\frac{1}{2}\left\{\left(\frac{\partial}{\partial x} \frac{\psi^{\prime}}{\rho_{0}{ }^{1 / 2}}\right)^{2}-\left(\frac{\partial}{\partial z} \frac{\psi^{\prime}}{\rho_{0}{ }^{1 / 2}}\right)^{2}\right. \\
\left.+\left[\frac{1}{2 \rho_{0}} \frac{d^{2} \rho_{0}}{d z^{2}}-\frac{3}{4 \rho_{0}{ }^{2}}\left(\frac{d \rho_{0}}{d z}\right)^{2}\right] \frac{\left(\psi^{\prime}\right)^{2}}{\rho_{0}}\right\}, \quad \\
F_{(z)}=-\left(\frac{\partial}{\partial x} \frac{\psi^{\prime}}{\rho_{0}{ }^{1 / 2}}\right)\left(\frac{\partial}{\partial z} \frac{\psi^{\prime}}{\rho_{0}{ }^{1 / 2}}\right) .
\end{gathered}
$$

The fact that (6.15) and (6.19) satisfy the group-velocity relation (2.16) is demonstrated for the reader in appendix $B$.

Under the Boussinesq approximation, we have the corresponding result

$$
\begin{gathered}
F_{(x)}=-\frac{g \bar{u}}{N^{2}} \rho^{\prime} \omega^{\prime}+\frac{g^{2}}{2 \rho_{r} N^{2}}\left(1-\frac{\bar{u}}{N^{2}} \frac{d \bar{\omega}}{d z}\right)\left(\rho^{\prime}\right)^{2} \\
-\frac{\rho_{r}}{2}\left\{\left(\frac{\partial \psi^{\prime}}{\partial x}\right)^{2}-\left(\frac{\partial \psi^{\prime}}{\partial z}\right)^{2}\right\}, \\
F_{(z)}=-\rho_{r} \frac{\partial \psi^{\prime}}{\partial x} \frac{\partial \psi^{\prime}}{\partial z} .
\end{gathered}
$$

Again, apart from a factor of $\rho_{r}$ this is consistent with the flux in (5.19) of Shepherd (1990).

\section{d. Finite-amplitude local conservation law}

As in the case of pseudoenergy, the derivation of the finite-amplitude pseudomomentum flux has been included for the reader in appendix $\mathrm{C}$. The form of the flux that reduces to (6.19) at small amplitude and is, therefore, consistent with (2.16) is

$$
\begin{gathered}
F_{(x)}=\left(\bar{u}+u^{\prime}\right) A+\frac{g}{\theta_{0}} \int_{0}^{\theta^{\prime}}\left\{\int_{\bar{\theta}}^{\bar{\theta}+\eta} R(\xi) d \xi\right\} d \eta \\
-\frac{1}{2}\left\{\left(\frac{\partial}{\partial x} \frac{\psi^{\prime}}{\rho_{0}{ }^{1 / 2}}\right)^{2}-\left(\frac{\partial}{\partial z} \frac{\psi^{\prime}}{\rho_{0}^{1 / 2}}\right)^{2}\right. \\
\left.+\left[\frac{1}{2 \rho_{0}} \frac{d^{2} \rho_{0}}{d z^{2}}-\frac{3}{4 \rho_{0}{ }^{2}}\left(\frac{d \rho_{0}}{d z}\right)^{2}\right] \frac{\left(\psi^{\prime}\right)^{2}}{\rho_{0}}\right\}, \\
F_{(z)}=w^{\prime} A-\left(\frac{\partial}{\partial x} \frac{\psi^{\prime}}{\rho_{0}{ }^{1 / 2}}\right)\left(\frac{\partial}{\partial z} \frac{\psi^{\prime}}{\rho_{0}{ }^{1 / 2}}\right),
\end{gathered}
$$

where $R(\xi)$ is defined by (6.4) and $A$ by $(6.10)$. The finite-amplitude flux of pseudomomentum in the Boussinesq limit is then

$$
\begin{gathered}
F_{(x)}=\left(\bar{u}+u^{\prime}\right) A-g \int_{0}^{\rho^{\prime}}[\mathcal{Z}(\bar{\rho}+\tilde{\rho}) \\
-\mathcal{Z}(\bar{\rho})] d \tilde{\rho}-\frac{\rho_{r}}{2}\left\{\left(\frac{\partial \psi^{\prime}}{\partial x}\right)^{2}-\left(\frac{\partial \psi^{\prime}}{\partial z}\right)^{2}\right\}, \\
F_{(z)}=w^{\prime} A-\rho_{r} \frac{\partial \psi^{\prime}}{\partial x} \frac{\partial \psi^{\prime}}{\partial z},
\end{gathered}
$$

where $Z(\eta)$ is defined by (6.11) and $A$ by (6.14).

\section{Discussion}

The anelastic and Boussinesq systems of equations have played a prominent role in the formulation of numerical models designed to simulate mesoscale phenomena, such as deep moist convection, squall-line life cycles, cloud dynamics, and airflow over complex terrain. In order to derive some dynamical understanding of the wave-mean flow interactions that ensue in such simulation, it is desirable to have wave-activity conservation laws that are not tied to a WKB approximation, are expressed entirely in terms of Eulerian quantities, and generalize naturally to finite amplitude. In this paper, such expressions have been derived for the anelastic and Boussinesq systems in two spatial dimensions.

It is worth asking why one would choose to focus on two-dimensional rather than three-dimensional flow, since the latter is evidently more general than the former. There are two reasons for this. First, the Hamiltonian formulation of three-dimensional incompres- 
sible flow appears to require the theory of constrained Hamiltonian systems (e.g., see Abarbanel et al. 1986, pp. 383-384; Salmon 1988b), and this lies beyond the scope of the representation (2.1) and the associated theory presented in sections 2 and 4 . It may nevertheless be possible to derive wave-activity conservation laws directly if one knows the form of the various integral invariants, as Haynes (1988) did for the hydrostatic primitive equations, but in the absence of an underlying Hamiltonian structure one would not seem to be assured of success. A second, rather different reason for focusing on two-dimensional flow in this paper is that the resulting expressions, relying as they do on the vorticity/potential-temperature representation (3.18) -note in particular the form of the integral invariants in section $4 \mathrm{~b}$-are much simpler than those that would obtain in a three-dimensional analysis. Certainly two-dimensional modeling studies using the anelastic or Boussinesq equations are common enough that the present results should be of some intrinsic interest.

The conservation laws derived here are of the form (1.1). For nonparallel steady basic flows, the conserved wave activity is a form of disturbance pseudoenergy; when the steady flow is parallel, there is in addition a conservation law for the disturbance pseudomomentum. The location of the most important results is summarized for the reader in Table 1.

The small-amplitude forms of the wave-activity densities and fluxes derived here are expressed solely in terms of Eulerian variables (i.e., not in terms of particle displacements), which makes them readily calculable from model data. The same applies to their finite-amplitude counterparts, with the following caveat: the functions $\Psi(\eta), Z_{0}(\eta), R(\eta)$, and $S(\eta)$ that arise in the expressions will only be able to be evaluated from Eulerian information alone if they are monotonic. This should not be an especially onerous restriction in practice, since when the finite-amplitude forms of the conservation laws are employed, the basic flow is completely arbitrary, and may be chosen to guarantee monotonicity of the functions in question. On the other hand, given a modest amount of Lagrangian infor-

TABLE 1. Equation numbers corresponding to the various waveactivity densities and fluxes derived in this paper.

\begin{tabular}{lll}
\hline \hline & \multicolumn{1}{c}{ Anelastic } & Boussinesq \\
\hline $\begin{array}{l}\text { Pseudoenergy, } \\
\text { finite amplitude }\end{array}$ & $(5.20 \mathrm{a}),(5.29)$ & $(5.21 \mathrm{a}),(5.30)$ \\
$\begin{array}{c}\text { Pseudoenergy, } \\
\text { small amplitude }\end{array}$ & $(5.20 \mathrm{~b}),(5.22)$ & $(5.21 \mathrm{~b}),(5.23)$ \\
$\begin{array}{c}\text { Pseudomomentum, } \\
\text { finite amplitude }\end{array}$ & $(6.10),(6.21)$ & $(6.14),(6.22)$ \\
$\begin{array}{c}\text { Pseudomomentum, } \\
\text { small amplitude }\end{array}$ & $(6.15),(6.19)$ & $(6.16),(6.20)$ \\
\hline
\end{tabular}

mation about the flow one might be able to consider nonmonotonic functions. A discussion of this point appears in section 5 of McIntyre and Shepherd (1987).

Another point to be made about the present results is that the expressions involve no averaging. This highlights the fact that their validity is not restricted to WKB-like conditions. It also means that even the pseudomomentum conservation law should be useful in conditions that are not homogeneous in the downstream coordinate $x$, where there is a nontrivial flux $F_{(x)}$. This is in contrast with the usual $x$-averaged results, for which there is essentially no distinction (at least at small amplitude) between the vertical fluxes of momentum and pseudomomentum in the absence of rotation (see below). It is for problems that are inhomogeneous in $x$ that the formulas derived here would be expected to yield the most new insight.

It is important to note the connection between the new pseudomomentum and pseudoenergy conservation laws and earlier "wave action" conservation laws (Whitham 1965; Bretherton and Garrett 1968). The wave action is defined under WKB conditions (i.e., slowly varying basic state) for small-amplitude, monochromatic wave trains, and is equal to the wave energy, suitably averaged over phase, divided by the intrinsic frequency $(\langle E\rangle / \hat{\Omega})$. Under conservative conditions it satisfies a conservation law of the form

$$
\frac{\partial}{\partial t}\left(\frac{\langle E\rangle}{\hat{\Omega}}\right)+\nabla \cdot\left(\frac{\langle E\rangle}{\hat{\Omega}} \mathbf{c}_{g}\right)=0
$$

[Bretherton and Garrett 1968, Eq. (1.9)]. The existence of such a conservation law in the appropriate small-amplitude, WKB limit was explicitly demonstrated for stratified parallel shear flow in the Boussinesq approximation by Garrett (1968), where

$$
E=\frac{\rho_{r}}{2}\left|\mathbf{v}^{\prime}\right|^{2}+\frac{1}{2} \frac{g^{2}}{N^{2} \rho_{r}} \rho^{\prime 2} ;
$$

the corresponding expression for $E$ in the anelastic case is given by

$$
E=-\frac{1}{2} \psi^{\prime} \omega^{\prime}+\frac{1}{2} \frac{\rho_{0} g^{2}}{N^{2} \theta_{0}^{2}} \theta^{\prime 2}
$$

Under such conditions it may be easily verified that the pseudoenergy and pseudomomentum invariants are proportional to the wave action, having the values $\Omega\langle E\rangle / \hat{\Omega}$ and $k\langle E\rangle / \hat{\Omega}$, respectively (where $k$ is the $x$ wavenumber and $\Omega$ the absolute frequency, both of which are conserved for wave trains in a steady parallel flow).

The critical reader may well question the idealized nature of the dynamics considered here; namely, conservative (unforced, inviscid) flow subject to simple boundary conditions. One could, if desired, extend the 
conservation laws derived here to include forced dissipative effects as a source-sink term on the right-hand side of (1.1), as Haynes (1988) has shown is generally possible. However, for essentially inviscid phenomena such as hydrodynamical instabilities or wave breaking, one would presumably be content with calculating these effects as a residual. With regard to the boundary conditions, they do not arise at all in the local form of the conservation law (1.1); one need only consider the boundary conditions when discussing global quantities. Hence, one may use (1.1) as a diagnostic for problems that are nonperiodic in $x$ and for which the component of $\mathbf{F}$ normal to the lower (or even upper) boundary does not vanish, so that there is no global conservation of wave activity. Indeed, the local form of the conservation law provides a concise way of representing the role of external influences on the dynamics.

We conclude by speculating about the possible uses of the conservation laws derived here as diagnostics of dynamical phenomena. One use is to characterize the structure and growth mechanisms of normal-mode instabilities. Whenever the dynamics of an instability is such that wave activity is globally conserved-this will always be the case for a local instability, but one must be careful about boundary conditions for global instabilities-it follows that a growing normal mode must have zero total wave activity, and will therefore usually consist of disturbance modes having opposite signs of wave activity. This sort of viewpoint has been exploited previously, with effect, to examine the instability of stratified shear flows (Cairns 1979; Ripa 1990), irrotational water waves (MacKay and Saffman 1986), and parallel flows in the shallow-water equations (Hayashi and Young 1987). It has also been used by Acheson (1976) to understand the overreflection of hydromagnetic internal gravity waves incident on a vortex-current sheet.

Another possible use is to quantify the drag associated with gravity-wave breaking. For a complete understanding of the interaction of waves and mean flows one requires a self-consistent theoretical framework consisting of a wave-activity conservation law of the form (1.1) together with an expression for the effect of the waves on the mean flow. For two-dimensional, nonrotating, stratified flow under conditions homogeneous in $x$, the $x$ average of the $x$ component of the momentum equation (3.9) is well defined and leads to the simple relation

$$
\rho_{0} \bar{u}_{t}=-\frac{\partial}{\partial z}\left(\rho_{0} \overline{u^{\prime} w^{\prime}}\right)=\frac{\partial}{\partial z}\left(\frac{1}{\rho_{0}} \overline{\frac{\partial \psi^{\prime}}{\partial z} \frac{\partial \psi^{\prime}}{\partial x}}\right),
$$

where now the overbar denotes the $x$ average, and the primes the departure therefrom. This expresses the wellknown effect of the vertical Reynolds stress divergence to drive horizontal flows. On the other hand, using (6.19) the $x$ average of the small-amplitude pseudomomentum conservation law takes the form

$$
\frac{\partial \bar{A}}{\partial t}=-\frac{\partial \bar{F}_{(z)}}{\partial z}=\frac{\partial}{\partial z}\left(\frac{1}{\rho_{0}} \overline{\frac{\partial \psi^{\prime}}{\partial z} \frac{\partial \psi^{\prime}}{\partial x}}-\frac{1}{2 \rho_{0}^{2}} \frac{d \rho_{0}}{d z} \overline{\psi^{\prime} \frac{\partial \psi^{\prime}}{\partial x}}\right)
$$

However, the second term in the final expression on the right-hand side of (7.5) vanishes under the $x$ average, leaving the result

$$
\rho_{0} \bar{u}_{t}=-\frac{\partial \bar{F}_{(z)}}{\partial z}=\frac{\partial \bar{A}}{\partial t},
$$

which is a concise statement of wave-mean flow interaction. The use of this sort of relation in understanding the driving of mean flows by gravity-wave transience and dissipation has been discussed by McIntyre (1973) and Grimshaw (1975), among others. In three-dimensional problems involving rotation, the situation is more complicated, but an analog of (7.6) nevertheless remains [e.g., see McIntyre (1977) and Andrews (1980)].

Unfortunately, the satisfactory situation represented by (7.6) has not yet been extended to the most general circumstances. For instance, when finite-amplitude effects are included, (7.4) remains as it is but the pseudomomentum flux $F_{(z)}$ includes an additional term $w^{\prime} A$, and this breaks the connection between waveactivity convergence and mean-flow driving. Also, even for small-amplitude disturbances the tight relationship of (7.6) depends upon most terms in the momentum budget vanishing under the $x$ average, and will therefore break down when the problem is inhomogeneous in $x$. It must be stressed that the wave-activity conservation laws derived here nevertheless remain valid, and express the torque exerted on the large-scale flow by the waves; however, we still lack a general theory for how that torque manifests itself in the mean-flow response.

A final possible use of these formulas that we will mention is the comparison of the small-amplitude with the finite-amplitude forms of the conservation laws in numerical simulations. This should provide a sensitive criterion for determining whether the essential dynamics of a given phenomenon is fundamentally linear or nonlinear. In particular, in the case of an alleged normal-mode instability, one could test the hypothesis by comparing the structure of the wave-activity flux divergence pattern for the growing normal mode with that realized in the nonlinear simulation. This sort of analysis is used extensively in Scinocca (1991), and will be presented in future publications.

Acknowledgments. Research support has been provided in the form of research grants to TGS from the Natural Sciences and Engineering Research Council and the Atmospheric Environment Service of Canada, and an NSERC postgraduate fellowship to JFS. 


\section{APPENDIX A \\ Jacobi's Identity}

Here we establish Jacobi's identity (2.7) for the $J$ matrix (4.5), which is representative of the anelastic system. In order to simplify the notation, we shall let

$$
f=\frac{\delta \mathcal{F}}{\delta U}, \quad g=\frac{\delta \mathcal{S}}{\delta U}, \quad \text { and } \quad k=\frac{\delta \mathcal{K}}{\delta U} .
$$

In terms of the inner product defined by (2.3), Jacobi's identity may be written

$$
\left(\frac{\delta}{\delta U}(f, J g), J k\right)+\mathrm{cyc}=0
$$

where "cyc" denotes all cyclic permutations of the expression. Now the first term in (A2) may be written

$$
\begin{aligned}
\left(\left(\frac{\delta f}{\delta U}, J g\right), J k\right)+\left(\left(f, \frac{\delta J}{\delta U} g\right), J k\right) \\
+\left(\left(f, J \frac{\delta g}{\delta U}\right), J k\right) .
\end{aligned}
$$

Because of the skew-symmetry condition (2.5), the first and third terms in (A3) when taken together with their cyclic permutations are identically equal to zero (cf. Morrison 1982), so that Jacobi's identity reduces to

$$
\left(\left(f, \frac{\delta J}{\delta U} g\right), J k\right)+\mathrm{cyc}=0
$$

Since the functional derivatives of $f, g$, and $k$ have now been taken care of, we may without loss of generality take

$$
\frac{\delta f}{\delta U}=0, \quad \frac{\delta g}{\delta U}=0, \quad \frac{\delta k}{\delta U}=0
$$

in what follows, in which case the first term in (A4) may be written as

$$
\left(f, \frac{\delta J}{\delta U} g\right)=\frac{\delta}{\delta U}(f, J g)=\frac{\delta}{\delta U} \iint f_{i} J_{i j} g_{i} d x d z,
$$

where the subscript 1 refers to the $\omega$ component while 2 refers to the $\theta$ component. For the particular form (4.5) of $J$, we may evaluate (A6) as follows:

$$
\begin{aligned}
& \frac{\delta}{\delta U} \iint\left[f_{1} \partial\left(g_{1}, \frac{\omega}{\rho_{0}}\right)\right. \\
&\left.+f_{1} \partial\left(\frac{g_{2}}{\rho_{0}}, \theta\right)+\frac{f_{2}}{\rho_{0}} \partial\left(g_{1}, \theta\right)\right] d x d z \\
&=\frac{\delta}{\delta U} \iint\left[\frac{\omega}{\rho_{0}} \partial\left(f_{1}, g_{1}\right)+\theta\left\{\partial\left(f_{1}, \frac{g_{2}}{\rho_{0}}\right)\right.\right. \\
&\left.\left.+\partial\left(\frac{f_{2}}{\rho_{0}}, g_{1}\right)\right\}\right] d x d z \quad \text { (A7) }
\end{aligned}
$$

$$
\equiv \frac{\delta(*)}{\delta U}=\left[\frac{1}{\rho_{0}} \partial\left(f_{1}, g_{1}\right), \partial\left(f_{1}, \frac{g_{2}}{\rho_{0}}\right)+\partial\left(\frac{f_{2}}{\rho_{0}}, g_{1}\right)\right] .
$$

The equality (A7) is a result of an integration by parts. We have, then, that (A4) may be written

$$
\begin{gathered}
\left(\frac{\delta(*)}{\delta U}, J k\right)+\operatorname{cyc}=\iint\left[\frac { \delta ( * ) } { \delta \omega } \left\{\partial\left(k_{1}, \frac{\omega}{\rho_{0}}\right)\right.\right. \\
\left.\left.+\partial\left(\frac{k_{2}}{\rho_{0}}, \theta\right)\right\}+\frac{1}{\rho_{0}} \frac{\delta(*)}{\delta \theta} \partial\left(k_{1}, \theta\right)+\operatorname{cyc}\right] d x d z \\
=\iint\left[\frac{\omega}{\rho_{0}} \partial\left(\frac{\delta(*)}{\partial \omega}, k_{1}\right)+\theta\left\{\partial\left(\frac{\delta(*)}{\delta \omega}, \frac{k_{2}}{\rho_{0}}\right)\right.\right. \\
\left.\left.+\partial\left(\frac{\delta(*)}{\delta \theta}, k_{1}\right)\right\}+\operatorname{cyc}\right] d x d z,
\end{gathered}
$$

where the last equality is the result of an integration by parts. It remains to show that the terms in the integrand of (A9) are zero. Toward this end, consider the term multiplying $\omega / \rho_{0}$ in (A9). Using (A8), this term plus its cyclic permutations may be written

$$
\begin{aligned}
& \frac{1}{\rho_{0}}\left[\partial\left(\partial\left(f_{1}, g_{1}\right), k_{1}\right)+\partial\left(\partial\left(g_{1}, k_{1}\right), f_{1}\right)\right. \\
& \left.\quad+\partial\left(\partial\left(k_{1}, f_{1}\right), g_{1}\right)\right]+\partial\left(f_{1}, g_{1}\right) \partial\left(\frac{1}{\rho_{0}}, k_{1}\right) \\
& \quad+\partial\left(g_{1}, k_{1}\right) \partial\left(\frac{1}{\rho_{0}}, f_{1}\right)+\partial\left(k_{1}, f_{1}\right) \partial\left(\frac{1}{\rho_{0}}, g_{1}\right) .
\end{aligned}
$$

Now the sum of the three terms in the square brackets is zero because it satisfies Jacobi's identity for partial derivatives,

$$
\partial(\partial(A, B), C)+\mathrm{cyc}=0 .
$$

It may be easily verified, upon expansion of the Jacobians, that the remaining terms in (A10) also sum to zero.

Consider now the terms multiplying $\theta$ in (A9). These terms may be written:

$$
\begin{aligned}
\frac{1}{\rho_{0}^{2}} & {\left[\partial\left(\partial\left(f_{1}, g_{1}\right), k_{2}\right)+\partial\left(\partial\left(f_{1}, g_{2}\right), k_{1}\right)\right.} \\
& \left.+\partial\left(\partial\left(f_{2}, g_{1}\right), k_{1}\right)+\mathrm{cyc}\right] \\
& +\frac{1}{\rho_{0}}\left[k_{2} \partial\left(\partial\left(f_{1}, g_{1}\right), \frac{1}{\rho_{0}}\right)+f_{2} \partial\left(\partial\left(\frac{1}{\rho_{0}}, g_{1}\right), k_{1}\right)\right. \\
& \left.+g_{2} \partial\left(\partial\left(f_{1}, \frac{1}{\rho_{0}}\right), k_{1}\right)+\mathrm{cyc}\right] \\
& +\frac{1}{\rho_{0}}\left[\partial\left(f_{1}, g_{1}\right) \partial\left(\frac{1}{\rho_{0}}, k_{2}\right)+\partial\left(f_{1}, g_{2}\right) \partial\left(\frac{1}{\rho_{0}}, k_{1}\right)\right.
\end{aligned}
$$




$$
\begin{gathered}
+\partial\left(g_{2}, k_{1}\right) \partial\left(\frac{1}{\rho_{0}}, f_{1}\right)+\partial\left(f_{2}, g_{1}\right) \partial\left(\frac{1}{\rho_{0}}, k_{1}\right) \\
+\partial\left(f_{2}, k_{1}\right) \partial\left(\frac{1}{\rho_{0}}, g_{1}\right)+\partial\left(f_{1}, g_{2}\right) \partial\left(\frac{1}{\rho_{0}}, k_{1}\right) \\
\left.+\partial\left(f_{2}, g_{1}\right) \partial\left(\frac{1}{\rho_{0}}, k_{1}\right)+\operatorname{cyc}\right]
\end{gathered}
$$

Each of the three terms in the first set of square brackets in (A12), together with their respective cyclic permutations, satisfies (A11). Therefore, the first two lines of (A12) are identically equal to zero. If we cyclically permute the indices of the explicit terms in the second set of square brackets so that they are all in terms of $k_{2}, f_{1}$, and $g_{1}$, then we have

$$
\begin{aligned}
k_{2} \partial\left(\partial\left(f_{1}, g_{1}\right), \frac{1}{\rho_{0}}\right)+k_{2} \partial\left(\partial\left(\frac{1}{\rho_{0}}, f_{1}\right), g_{1}\right) \\
+k_{2} \partial\left(\partial\left(g_{1}, \frac{1}{\rho_{0}}\right), f_{1}\right)+\text { cyc. }
\end{aligned}
$$

An expansion of the Jacobians in the above expression reveals that these three terms, and therefore their cyclic permutations, sum to zero.

If we again cyclically permute the indices of the explicit terms in the final set of square brackets so that they are all in terms of $k_{2}, f_{1}$, and $g_{1}$, then (after some cancellation) we have

$$
\begin{aligned}
\partial\left(f_{1}, g_{1}\right) \partial\left(\frac{1}{\rho_{0}}, k_{2}\right)+\partial( & \left.g_{1}, k_{2}\right) \partial\left(\frac{1}{\rho_{0}}, f_{1}\right) \\
& +\partial\left(k_{2}, f_{1}\right) \partial\left(\frac{1}{\rho_{0}}, g_{1}\right)+\text { cyc. }
\end{aligned}
$$

It can be easily verified that these terms, and therefore their cyclic permutations, sum to zero. We have then that Jacobi's identity is satisfied for $J$ of the form (4.5) associated with the anelastic system.

\section{APPENDIX B}

\section{Group-Velocity Property}

In this appendix we shall demonstrate that the condition (2.16) is satisfied by the small-amplitude waveactivity densities and fluxes presented for the cases of pseudoenergy and pseudomomentum in this paper.

\section{Pseudoenergy}

In order to determine the dispersion relation for the anelastic system we let

$$
\psi^{\prime}=\rho_{0}{ }^{1 / 2} \phi^{\prime}
$$

We have then that (3.17) may be written

$$
\omega^{\prime}=\frac{\gamma(z)}{\rho_{0}^{1 / 2}} \phi^{\prime}+\frac{1}{\rho_{0}^{1 / 2}} \nabla^{2} \phi^{\prime}
$$

where

$$
\gamma(z)=-\frac{3}{4 \rho_{0}^{2}}\left(\frac{d \rho_{0}}{d z}\right)^{2}+\frac{1}{2 \rho_{0}} \frac{d^{2} \rho_{0}}{d z^{2}},
$$

while (5.15) take the form

$$
\begin{aligned}
& \omega_{t}^{\prime}=-\partial\left(\rho_{0}{ }^{1 / 2} \phi^{\prime}, \frac{\bar{\omega}}{\rho_{0}}\right)-\partial\left(\bar{\psi}, \frac{\omega^{\prime}}{\rho_{0}}\right)-\frac{g}{\theta_{0}} \frac{\partial \theta^{\prime}}{\partial x}, \\
& \theta_{t}^{\prime}=-\frac{1}{\rho_{0}} \partial\left(\rho_{0}{ }^{1 / 2} \phi^{\prime}, \bar{\theta}\right)-\frac{1}{\rho_{0}} \partial\left(\bar{\psi}, \theta^{\prime}\right) .
\end{aligned}
$$

If we assume a scale separation between the disturbances and the mean flow, then solutions to (B2), (B4), and (B5) may be sought using the WKB ansatz

$$
\begin{aligned}
& \phi^{\prime}=\mathscr{R}\left\{\Phi(X, Z) e^{\left[i \mu^{-1} \Lambda(X, Z)-i \Omega t\right]}\right\}, \\
& \theta^{\prime}=\mathcal{R}\left\{\Theta(X, Z) e^{\left[i \mu^{-1} \Lambda(X, Z)-i \Omega t\right]}\right\},
\end{aligned}
$$

where

$(X, Z)=\mu(x, z), \quad \mu=\frac{\text { length scale of perturbations }}{\text { length scale of steady-state fiow }}$.

Substituting (B6) into (B2), (B4), and (B5), and retaining terms to zeroeth order in $\mu$ results in the dispersion relation

$$
\begin{aligned}
& (\Omega-\mathbf{k} \cdot \overline{\mathbf{v}})\left[\left(\gamma(z)-k^{2}-m^{2}\right)(\Omega-k \bar{u}\right. \\
& \left.-m \bar{w}\left\{1-i \frac{3}{2 m \rho_{0}} \frac{d \rho_{0}}{d z}\right\}\right)+\rho_{0} k \partial_{z}\left(\frac{\bar{\omega}}{\rho_{0}}\right) \\
& \left.-\rho_{0} m \partial_{x}\left(\frac{\bar{\omega}}{\rho_{0}}\right)\left(1-i \frac{1}{2 m \rho_{0}} \frac{d \rho_{0}}{d z}\right)+i \frac{1}{\rho_{0}} \partial(\bar{\psi}, \gamma(z))\right] \\
& \quad+k^{2} N_{z}^{2}-k m N_{x}^{2}\left(1+i \frac{1}{2 m \rho_{0}} \frac{d \rho_{0}}{d z}\right)=0,
\end{aligned}
$$

where $\mathbf{k} \equiv(k, m)$. In obtaining (B8) we have used the definitions

$$
\begin{aligned}
(k, m) & =\left(\frac{\partial \Lambda}{\partial x}, \frac{\partial \Lambda}{\partial z}\right), \\
N_{\alpha}{ }^{2} & =\frac{g}{\theta_{0}} \frac{\partial \bar{\theta}}{\partial \alpha}, \quad \alpha \in(x, z) .
\end{aligned}
$$

For basic flows that are independent of $x,(\mathrm{~B} 8)$ is purely real and we need impose no restriction on the perturbations other than $\mu \ll 1$. When the basic flow is nonparallel, on the other hand, (B8) contains some imag- 
inary terms. Three of these terms may be eliminated if we restrict the vertical wavenumber according to

$$
m \gg \frac{3}{2} \frac{1}{\rho_{0}} \frac{d \rho_{0}}{d z} .
$$

This condition was discussed in section 5 and is identical to (5.19). The final imaginary term involves the vertical derivative of $\gamma(z)$, and may be neglected against $\gamma m \bar{w}$ provided

$$
m \gg \frac{1}{\gamma} \frac{d \gamma}{d z} .
$$

Insofar as the term on the right-hand side of (B9b) is proportional to the rate at which the density scale height varies in the vertical, the condition (B9b) is not particularly restrictive given the primary WKB ansatz $\mu$ $\ll 1$. We shall therefore neglect the imaginary terms in (B8) from now on, bearing in mind that for nonparallel basic flows the group-velocity relation (2.16) will only hold under the restrictions (B9).

For notational convenience we define

$$
\begin{aligned}
& \hat{\Omega}=\Omega-\mathbf{k} \cdot \overline{\mathbf{v}}, \\
& \hat{\omega}=k \partial_{z} \frac{\bar{\omega}}{\rho_{0}}-m \partial_{x} \frac{\bar{\omega}}{\rho_{0}}, \\
& \hat{N}=k N_{z}^{2}-m N_{x}^{2}, \\
& \hat{\gamma}=k^{2}+m^{2}+\gamma(z) .
\end{aligned}
$$

Using this notation, the dispersion relation may be written

$$
\hat{\gamma} \hat{\Omega}^{2}=\rho_{0} \hat{\omega} \hat{\Omega}+k \hat{N} .
$$

In the derivation that follows, for the general case of nonparallel flow under the approximation (B9), it is clear that we need not include $\gamma(z)$ in the definition of $\hat{\gamma}(z)$ since it is formally negligible. However, the inclusion of this term extends the derivation to also include the most general case of parallel flow in which no such approximation is necessary.

The group velocity may now be easily obtained from (B11). For this particular dispersion relation we have

$$
\begin{aligned}
c_{g x} & =\frac{\partial \Omega}{\partial k}=\left(\rho_{0} \hat{\omega} \hat{\Omega}+2 k \hat{N}\right)^{-1}\left[-2 k \hat{\Omega}^{3}\right. \\
+ & \left.\rho_{0} \partial_{z}\left(\bar{\omega} / \rho_{0}\right) \hat{\Omega}^{2}+\left(k N_{z}^{2}+\hat{N}+\rho_{0} \bar{u} \hat{\omega}\right) \hat{\Omega}+2 k \bar{u} \hat{N}\right], \\
c_{g z} & =\frac{\partial \Omega}{\partial m}=\left(\rho_{0} \hat{\omega} \hat{\Omega}+2 k \hat{N}\right)^{-1}\left[-2 m \hat{\Omega}^{3}\right. \\
& \left.-\rho_{0} \partial_{x}\left(\bar{\omega} / \rho_{0}\right) \hat{\Omega}^{2}+\left(-k N_{x}^{2}+\rho_{0} \bar{w} \hat{\omega}\right) \hat{\Omega}+2 k \bar{w} \hat{N}\right] .
\end{aligned}
$$

In deriving (B12) and (B13) we have used the dispersion relation (B11) to eliminate $\hat{\gamma}$ from the final forms.
In satisfying the relation $(2.16)$ we need to calculate the average over phase of the disturbance fields in the wave-activity density and flux. This average is denoted by angle brackets. In performing these averages we have to evaluate the term $\left\langle\psi^{\prime} \psi^{\prime}\right\rangle$. This is determined to be

$$
\left\langle\psi^{\prime} \psi^{\prime}\right\rangle=\rho_{0}\left\langle\phi^{\prime} \phi^{\prime}\right\rangle=\frac{1}{2} \rho_{0} \mathcal{R}\left\{\Phi^{\prime *} \Phi^{\prime}\right\}=\frac{1}{2} \rho_{0}|\Phi|^{2} .
$$

As a result one finds

$$
\begin{gathered}
\left\langle\theta^{\prime} \psi^{\prime}\right\rangle=-\frac{1}{2} \frac{\theta_{0}}{g} \frac{\hat{N}}{\hat{\Omega}}|\Phi|^{2}, \\
\left\langle\omega^{\prime} \psi^{\prime}\right\rangle=-\frac{1}{2} \rho_{0}\left(\frac{\hat{\omega}}{\hat{\Omega}}+\frac{k \hat{N}}{\rho_{0} \hat{\Omega}^{2}}\right)|\Phi|^{2}, \\
\left\langle\theta^{\prime} \omega^{\prime}\right\rangle=\frac{1}{2} \frac{\theta_{0}}{g} \frac{\hat{N}}{\hat{\Omega}}\left(\frac{\hat{\omega}}{\hat{\Omega}}+\frac{k \hat{N}}{\rho_{0} \hat{\Omega}^{2}}\right)|\Phi|^{2} .
\end{gathered}
$$

In (B14) we have used the relations

$$
\begin{aligned}
& \theta^{\prime}=-\frac{\theta_{0}}{g \rho_{0}} \frac{\hat{N}}{\hat{\Omega}} \psi^{\prime}, \\
& \omega^{\prime}=-\left(\frac{\hat{\omega}}{\hat{\Omega}}+\frac{k \hat{N}}{\rho_{0} \hat{\Omega}^{2}}\right) \psi^{\prime},
\end{aligned}
$$

which were determined from the linearized equations in accord with (B9).

Given (B14) we may now evaluate the average over phase of the wave activity $(5.20 \mathrm{~b})$. This calculation results in the expression

$$
\begin{aligned}
\langle A\rangle= & \frac{|\Phi|^{2}}{4 N_{z}{ }^{4} \hat{\Omega}^{3}}\left[\hat{\Omega}^{2}\left(\rho_{0} N_{z}{ }^{4} \hat{\omega}\right)+\hat{\Omega}\left(k N_{z}{ }^{4} \hat{N}\right.\right. \\
& +2 \rho_{0} \bar{u} N_{z}{ }^{2} \hat{N} \hat{\omega}+N_{z}{ }^{2} \hat{N}^{2} \\
& \left.\left.-\rho_{0} \bar{u} \partial_{z}\left(\bar{\omega} / \rho_{0}\right) \hat{N}^{2}\right)+2 k \bar{u} N_{z}{ }^{2} \hat{N}^{2}\right] .
\end{aligned}
$$

Similar averages over $F_{(x)},(5.22 \mathrm{a})$, and $F_{(z)},(5.22 \mathrm{~b})$, result in

$$
\begin{aligned}
& \left\langle F_{(x)}\right\rangle=\frac{|\Phi|^{2}}{4 N_{z}{ }^{4} \hat{\Omega}^{3}}\left[\hat{\Omega}^{4}\left(-2 k N_{z}{ }^{4}\right)+\hat{\Omega}^{3}\left(\rho_{0} N_{z}{ }^{4} \partial_{z}\left(\bar{\omega} / \rho_{0}\right)\right.\right. \\
& \left.-2 k^{2} \bar{u} N_{z}{ }^{4}-2 k m \bar{w} N_{z}{ }^{4}\right)+\hat{\Omega}^{2}\left(2 \rho_{0} \bar{u} N_{z}{ }^{4} \hat{\omega}+2 N_{z}{ }^{4} \hat{N}\right) \\
& +\hat{\Omega}\left(2 k \bar{u} N_{z}{ }^{4} \hat{N}+2 \rho_{0} \bar{u}^{2} N_{z}{ }^{2} \hat{N} \hat{\omega}+2 \bar{u} N_{z}{ }^{2} \hat{N}^{2}\right. \\
& \left.\left.\left.\quad-\rho_{0} \bar{u}^{2} \partial_{z}\left(\bar{\omega} / \rho_{0}\right) \hat{N}^{2}\right)+2 k \bar{u}^{2} N_{z}{ }^{2} \hat{N}^{2}\right], \quad \text { (B18a }\right) \\
& \left\langle F_{(z)}\right\rangle=\frac{|\Phi|^{2}}{4 N_{z}{ }^{4} \hat{\Omega}^{3}}\left[\hat{\Omega}^{4}\left(-2 m N_{z}{ }^{4}\right)\right. \\
& +\hat{\Omega}^{3}\left(-\rho_{0} \partial_{x}\left(\bar{\omega} / \rho_{0}\right) N_{z}{ }^{4}-2 k m \bar{u} N_{z}{ }^{4}-2 m^{2} \bar{w} N_{z}{ }^{4}\right) \\
& +\hat{\Omega}^{2}\left(2 \rho_{0} \bar{w} N_{z}{ }^{4} \hat{\omega}\right)+\hat{\Omega}\left(2 k \bar{w} N_{z}{ }^{4} \hat{N}-2 \rho_{0} \bar{u}^{2} N_{x}{ }^{2} \hat{N} \hat{\omega}\right. \\
& \left.\left.\quad+\rho_{0} \bar{u}^{2} \partial_{x}\left(\bar{\omega} / \rho_{0}\right) \hat{N}^{2}\right)-2 k \bar{u}^{2} N_{x}{ }^{2} \hat{N}^{2}\right] . \quad(\mathrm{B} 18 \mathrm{~b})
\end{aligned}
$$


We first consider the $z$ component of (2.16) and evaluate

$$
\left\langle F_{(z)}\right\rangle-c_{g z}\langle A\rangle
$$

If we multiply this expression by $\rho_{0} \hat{\omega} \hat{\Omega}+2 k \hat{N}$ and use (B13), (B17), and (B18b) it is found that, after some cancellation, (B19) reduces to

$$
\begin{aligned}
\hat{\Omega}^{4}( & -2 k m N_{z}{ }^{4} \hat{N}-2 \rho_{0} k m \bar{u} N_{z}{ }^{4} \hat{\omega} \\
& -2 \rho_{0} m^{2} \bar{w} N_{z}{ }^{4} \hat{\omega}+4 \rho_{0} m \bar{u} N_{z}{ }^{2} \hat{N} \hat{\omega} \\
& \left.+2 m N_{z}{ }^{2} \hat{N}^{2}-2 \rho_{0} m \bar{u} \partial_{z}\left(\bar{\omega} / \rho_{0}\right) \hat{N}^{2}\right) \\
+ & \hat{\Omega}^{3}\left(\rho_{0}{ }^{2} \bar{w} N_{z}{ }^{4} \hat{\omega}^{2}-\rho_{0} k \partial_{x}\left(\bar{\omega} / \rho_{0}\right) N_{z}{ }^{4} \hat{N}\right. \\
& -4 m k^{2} \bar{u} N_{z}{ }^{4} \hat{N}-4 m^{2} k \bar{w} N_{z}{ }^{4} \hat{N}+4 m k \bar{u} N_{z}{ }^{2} \hat{N}^{2} \\
& +2 \rho_{0}{ }^{2} \bar{u} \partial_{x}\left(\bar{\omega} / \rho_{0}\right) N_{z}{ }^{2} \hat{N} \hat{\omega}+\rho_{0} \partial_{x}\left(\bar{\omega} / \rho_{0}\right) N_{z}{ }^{2} \hat{N}^{2} \\
& \left.-\rho_{0}{ }^{2} \partial_{x}\left(\bar{\omega} / \rho_{0}\right) \partial_{z}\left(\bar{\omega} / \rho_{0}\right) \hat{N}^{2}+\rho_{0} k N_{x}{ }^{2} N_{z}{ }^{4} \hat{\omega}\right) \\
+ & \hat{\Omega}^{2}\left(3 \rho_{0} k \bar{w} N_{z}{ }^{4} \hat{N} \hat{\omega}-2 \rho_{0}{ }^{2} \bar{u}^{2} N_{x}{ }^{2} \hat{N} \hat{\omega}^{2}\right. \\
& +\rho_{0}{ }^{2} \bar{u}^{2} \partial_{x}\left(\bar{\omega} / \rho_{0}\right) \hat{N}^{2} \hat{\omega}+2 \rho_{0} k \bar{u} \partial_{x}\left(\bar{\omega} / \rho_{0}\right) N_{z}{ }^{2} \hat{N}^{2} \\
& +k^{2} N_{x}{ }^{2} N_{z}{ }^{4} \hat{N}+2 \rho_{0} k \bar{u} N_{x}{ }^{2} N_{z}{ }^{2} \hat{N} \hat{\omega}+k N_{x}{ }^{2} N_{z}{ }^{2} \hat{N}^{2} \\
& -\rho_{0} k \bar{u} \partial_{z}\left(\bar{\omega} / \rho_{0}\right) N_{x}{ }^{2} \hat{N}^{2}-2 \rho_{0}{ }^{2} \bar{u} \bar{w} N_{z}{ }^{2} \hat{N} \hat{\omega}^{2} \\
& \left.-\rho_{0} \bar{w} N_{z}{ }^{2} \hat{N}^{2} \hat{\omega}+\rho_{0}{ }^{2} \bar{u} \bar{w} \partial_{z}\left(\bar{\omega} / \rho_{0}\right) \hat{N}^{2} \hat{\omega}\right) \\
+ & \hat{\Omega}\left(-6 \rho_{0} k \bar{u}^{2} N_{x}{ }^{2} \hat{N}^{2} \hat{\omega}+2 k^{2} \bar{w} N_{z}{ }^{4} \hat{N}^{2}\right. \\
& +2 \rho_{0} k \bar{u}^{2} \partial_{x}\left(\bar{\omega} / \rho_{0}\right) \hat{N}^{3}+2 k^{2} \bar{u} N_{x}{ }^{2} N_{z}{ }^{2} \hat{N}^{2} \\
& -2 \rho_{0} k \bar{u} \bar{w} N_{z}{ }^{2} \hat{N}^{2} \hat{\omega}-4 \rho_{0} k \bar{u} \bar{w} N_{z}{ }^{2} \hat{N}^{2} \hat{\omega} \\
& \left.-2 k \bar{w} N_{z}{ }^{2} \hat{N}^{3}+2 \rho_{0} k \bar{u} \bar{w} \partial_{z}\left(\bar{\omega} / \rho_{0}\right) \hat{N}^{3}\right) \\
& -\left(4 k^{2} \bar{u}^{2} N_{x}{ }^{2} \hat{N}^{3}+4 k^{2} \bar{u} \bar{w} N_{z}{ }^{2} \hat{N}^{3}\right)
\end{aligned}
$$

multiplied by the common factor

$$
\frac{|\Phi|^{2}}{4 N_{z}^{4} \hat{\Omega}^{3}}
$$

One can easily (but tediously) verify that each one of the coefficients in (B20) is identically equal to zero. To do this, it is necessary to make use of the two identities

$$
\begin{gathered}
\bar{w}=-\frac{N_{x}^{2}}{N_{z}^{2}} \bar{u}, \\
N_{x}^{2}=-\rho_{0} \bar{w} \partial_{z}\left(\bar{\omega} / \rho_{0}\right)-\rho_{0} \bar{u} \partial_{x}\left(\bar{\omega} / \rho_{0}\right),
\end{gathered}
$$

which are a consequence of the steady-state nature of the background fields as expressed in (3.22).

Forming now the $x$ counterpart to (B19) we have

$$
\left\langle F_{(x)}\right\rangle-c_{g x}\langle A\rangle \text {. }
$$

Again, multiplying this expression by $\rho_{0} \hat{\omega} \hat{\Omega}+2 k \hat{N}$, and using (B12), (B17), and (B18a), it is found that, after some cancellation, (B23) reduces to

$\hat{\Omega}^{4}\left(-2 \rho_{0} k^{2} \bar{u} N_{z}{ }^{4} \hat{\omega}-2 \rho_{0} k m \bar{w} N_{z}{ }^{4} \hat{\omega}-2 k^{2} N_{z}{ }^{4} \hat{N}\right.$

$$
\begin{aligned}
& +4 \rho_{0} k \bar{u} N_{z}{ }^{2} \hat{N} \hat{\omega}+2 k N_{z}{ }^{2} \hat{N}^{2} \\
& \left.-2 \rho_{0} k \bar{u} \partial_{z}\left(\bar{\omega} / \rho_{0}\right) \hat{N}^{2}\right) \\
+ & \hat{\Omega}^{3}\left(\rho_{0}{ }^{2} \bar{u} N_{z}{ }^{4} \hat{\omega}^{2}+\rho_{0} N_{z}{ }^{4} \hat{N} \hat{\omega}+\rho_{0} k N_{z}{ }^{4} \partial_{z}\left(\bar{\omega} / \rho_{0}\right) \hat{N}\right. \\
& -4 k^{3} \bar{u} N_{z}{ }^{4} \hat{N}-4 k^{2} m \bar{w} N_{z}{ }^{4} \hat{N}+4 k^{2} \bar{u} N_{z}{ }^{2} \hat{N}^{2} \\
& -2 \rho_{0}{ }^{2} \bar{u} \partial_{z}\left(\bar{\omega} / \rho_{0}\right) N_{z}{ }^{2} \hat{N} \hat{\omega}-\rho_{0} N_{z}{ }^{2} \partial_{z}\left(\bar{\omega} / \rho_{0}\right) \hat{N}^{2} \\
& \left.+\rho_{0}{ }^{2} \bar{u}\left(\partial_{z}\left(\bar{\omega} / \rho_{0}\right)\right)^{2} \hat{N}^{2}-\rho_{0} k N_{z}{ }^{6} \hat{\omega}\right) \\
+ & \hat{\Omega}^{2}\left(3 \rho_{0} k \bar{u} N_{z}{ }^{4} \hat{N} \hat{\omega}+2 k N_{z}{ }^{4} \hat{N}^{2}\right. \\
& -\rho_{0} k \bar{u} \partial_{z}\left(\bar{\omega} / \rho_{0}\right) N_{z}{ }^{2} \hat{N}^{2}-k^{2} N_{z}{ }^{6} \hat{N} \\
& -2 \rho_{0} \bar{u} k N_{z}{ }^{4} \hat{N} \hat{\omega}-N_{z}{ }^{2} \hat{N}^{3}+\rho_{0} \bar{u} \partial_{z}\left(\bar{\omega} / \rho_{0}\right) \hat{N}^{3} \\
& \left.\quad-\rho_{0} \bar{u} N_{z}{ }^{2} \hat{N}^{2} \hat{\omega}\right) . \quad \text { (B24) }
\end{aligned}
$$

Once again, a liberal use of (B21) and (B22) in (B24) demonstrates that the coefficient of each power of $\hat{\Omega}$ vanishes independently. Therefore, we have the result that the particular small-amplitude forms of $A$ (5.20b) and F (5.22) for pseudoenergy indeed satisfy the group-velocity condition (2.16).

\section{Pseudomomentum}

Since we are dealing with the conservation of $x$ momentum in this case, the steady-state flow is assumed to be parallel. As a consequence, we have the result that, with no restriction on $m$ (apart from $\mu \ll 1$ ), the dispersion relation (B8) reduces to

$$
\hat{\gamma} \hat{\Omega}^{2}=\rho_{0} k d_{z}\left(\bar{\omega} / \rho_{0}\right) \hat{\Omega}+k^{2} N_{z}^{2},
$$

where now $\bar{w}=0$ in the definition (B10a) of $\hat{\Omega}$. The group velocity in this case is then

$$
\begin{gathered}
c_{g x}=\left(\rho_{0} k d_{z}\left(\bar{\omega} / \rho_{0}\right) \hat{\Omega}+2 k^{2} N_{z}^{2}\right)^{-1}\left[-2 k \hat{\Omega}^{3}\right. \\
+\rho_{0} d_{z}\left(\bar{\omega} / \rho_{0}\right) \hat{\Omega}^{2}+\left(2 k N_{z}^{2}+\rho_{0} k \bar{u} d_{z}\left(\bar{\omega} / \rho_{0}\right)\right) \hat{\Omega} \\
\left.+2 k^{2} \bar{u} N_{z}^{2}\right],
\end{gathered}
$$

The average over phase of the wave activity $(6.15)$ is then

$$
\langle A\rangle=\frac{|\Phi|^{2}}{4 \hat{\Omega}^{3}}\left[\rho_{0} k^{2} d_{z}\left(\bar{\omega} / \rho_{0}\right) \hat{\Omega}+2 k^{3} N_{z}^{2}\right] .
$$

In a similar manner we have that the average over phase of the flux (6.19) is

$$
\begin{array}{r}
\left\langle F_{(x)}\right\rangle=\frac{|\Phi|^{2}}{4 \hat{\Omega}^{3}}\left[-2 k^{2} \hat{\Omega}^{3}+\rho_{0} k d_{z}\left(\bar{\omega} / \rho_{0}\right) \hat{\Omega}^{2}\right. \\
\left.+\left(2 k^{2} N_{z}^{2}+\rho_{0} k^{2} \bar{u} d_{z}\left(\bar{\omega} / \rho_{0}\right)\right) \hat{\Omega}+2 k^{3} \bar{u} N_{z}^{2}\right], \\
\left\langle F_{(z)}\right\rangle=\frac{|\Phi|^{2}}{4 \hat{\Omega}^{3}}\left(-2 k m \hat{\Omega}^{3}\right) .
\end{array}
$$


It is now a straightforward matter to show, by substitution of (B26), (B27), and (B28), that (2.16) is satisfied.

\section{APPENDIX C}

\section{Local Flux Forms}

In this appendix we provide the details of the calculation of finite-amplitude wave-activity flux in both the pseudoenergy and pseudomomentum cases.

\section{Pseudoenergy}

Here we provide a derivation of the expression (5.27) for the local time derivative of $A_{2}$. In order to cast the local time derivative of $A_{2}$ in flux form, we first consider the material derivative

$$
\begin{aligned}
\frac{D A_{2}}{D t}= & \frac{\partial A_{2}}{\partial \bar{\theta}} \frac{D \bar{\theta}}{D t}+\frac{\partial A_{2}}{\partial \theta^{\prime}} \frac{D \theta^{\prime}}{D t}+\frac{\partial A_{2}}{\partial \bar{\omega}} \frac{D \bar{\omega}}{D t} \\
& +\frac{\partial A_{2}}{\partial \omega^{\prime}} \frac{D \omega^{\prime}}{D t}+\frac{\partial A_{2}}{\partial \rho_{0}} \frac{D \rho_{0}}{D t} \\
= & \left(\frac{\partial A_{2}}{\partial \bar{\theta}}-\frac{\partial A_{2}}{\partial \theta^{\prime}}\right) \frac{D \bar{\theta}}{D t}+\left(\frac{\partial A_{2}}{\partial \bar{\omega}}-\frac{\partial A_{2}}{\partial \omega^{\prime}}\right) \frac{D \bar{\omega}}{D t} \\
& -\frac{\partial A_{2}}{\partial \omega^{\prime}}\left(\frac{\omega}{\rho_{0}^{2}} \frac{d \rho_{0}}{d z} \frac{\partial \psi}{\partial x}+\frac{g}{\theta_{0}} \frac{\partial \theta}{\partial x}\right) \\
& \quad-\frac{\partial A_{2}}{\partial \rho_{0}} \frac{1}{\rho_{0}} \frac{d \rho_{0}}{d z} \frac{\partial \psi}{\partial x},
\end{aligned}
$$

where

$$
\frac{D}{D t}=\frac{\partial}{\partial t}+\mathbf{v} \cdot \nabla
$$

In $(\mathrm{C} 1)$ we have used the fact that

$$
\frac{D \theta}{D t}=0 \Rightarrow \frac{D \theta^{\prime}}{D t}=-\frac{D \bar{\theta}}{D t}
$$

and

$$
\frac{D \omega^{\prime}}{D t}=-\frac{D \bar{\omega}}{D t}-\frac{\omega}{\rho_{0}^{2}} \frac{d \rho_{0}}{d z} \frac{\partial \psi}{\partial x}-\frac{g}{\theta_{0}} \frac{\partial \theta}{\partial x} .
$$

Now, in $(\mathrm{C} 1)$ we have

$$
\begin{aligned}
& \frac{D \bar{\theta}}{D t}=\frac{1}{\rho_{0}} \partial\left(\psi^{\prime}, \bar{\theta}\right), \\
& \frac{D \bar{\omega}}{D t}=\frac{1}{\rho_{0}} \partial(\psi, \bar{\omega}),
\end{aligned}
$$

$$
\begin{array}{r}
\frac{\partial A_{2}}{\partial \bar{\theta}}=\left(\bar{\omega}+\omega^{\prime}\right)\left[\Psi^{\prime}\left(\bar{\theta}+\theta^{\prime}\right)-\Psi^{\prime}(\bar{\theta})\right]-\bar{\omega} \Psi^{\prime \prime}(\bar{\theta}) \theta^{\prime} \\
-\rho_{0}\left[Z_{0}\left(\bar{\theta}+\theta^{\prime}\right)-Z_{0}(\bar{\theta})-Z_{0}^{\prime}(\bar{\theta}) \theta^{\prime}\right],
\end{array}
$$

$\frac{\partial A_{2}}{\partial \theta^{\prime}}=\left(\bar{\omega}+\omega^{\prime}\right) \Psi^{\prime}\left(\bar{\theta}+\theta^{\prime}\right)-\bar{\omega} \Psi^{\prime}(\bar{\theta})$

$$
-\rho_{0}\left[Z_{0}\left(\bar{\theta}+\theta^{\prime}\right)-Z_{0}(\bar{\theta})\right],
$$

$$
\begin{aligned}
& \frac{\partial A_{2}}{\partial \bar{\omega}}=\Psi\left(\bar{\theta}+\theta^{\prime}\right)-\Psi(\bar{\theta})-\Psi^{\prime}(\bar{\theta}) \theta^{\prime}, \\
& \frac{\partial A_{2}}{\partial \omega_{.}^{\prime}}=\Psi\left(\bar{\theta}+\theta^{\prime}\right)-\Psi(\bar{\theta}), \\
& \frac{\partial A_{2}}{\partial \rho_{0}}=-\int_{0}^{\theta^{\prime}}\left[Z_{0}(\bar{\theta}+\eta)-Z_{0}(\bar{\theta})\right] d \eta .
\end{aligned}
$$

A substitution of these expressions into ( $\mathrm{Cl}$ ) results in

$$
\begin{aligned}
& \frac{\partial A_{2}}{\partial t}=-\partial\left(\psi, \frac{A_{2}}{\rho_{0}}\right)+\left\{\left(\Psi\left(\bar{\theta}+\theta^{\prime}\right)-\Psi(\bar{\theta})-\Psi^{\prime}(\bar{\theta}) \theta^{\prime}\right)\right. \\
& \cdot\left[\bar{\omega} \partial\left(\bar{\psi}, \frac{1}{\rho_{0}}\right)+\frac{1}{\rho_{0}} \partial(\bar{\psi}, \bar{\omega})\right] \\
&\left.-\left(\Psi\left(\bar{\theta}+\theta^{\prime}\right)-\Psi(\bar{\theta})\right) \frac{g}{\theta_{0}} \frac{\partial \theta^{\prime}}{\partial x}\right\} \\
&+\left\{-\Psi^{\prime}(\bar{\theta}) \theta^{\prime}\left[\bar{\omega} \partial\left(\psi^{\prime}, \frac{1}{\rho_{0}}\right)+\frac{1}{\rho_{0}} \partial\left(\psi^{\prime}, \bar{\omega}\right)\right]\right. \\
&\left.+\frac{1}{\rho_{0}} \partial\left(\psi^{\prime}, \bar{\theta}\right)\left[-\bar{\omega} \Psi^{\prime \prime}(\bar{\theta}) \theta^{\prime}+\rho_{0} Z_{0}^{\prime}(\bar{\theta}) \theta^{\prime}\right]\right\} \\
&-\frac{\omega^{\prime}}{\rho_{0}} \Psi^{\prime}(\bar{\theta}) \partial\left(\psi^{\prime}, \bar{\theta}\right), \quad(\mathrm{C} 2)
\end{aligned}
$$

after using

$$
\begin{aligned}
\frac{D A_{2}}{D t} & =\frac{\partial A_{2}}{\partial t}+\frac{1}{\rho_{0}} \partial\left(\psi, A_{2}\right) \\
& =\frac{\partial A_{2}}{\partial t}+\partial\left(\psi, \frac{A_{2}}{\rho_{0}}\right)-A_{2} \partial\left(\psi, \frac{1}{\rho_{0}}\right) .
\end{aligned}
$$

Using the steady-state balance (3.22a), the first term in large braces on the right-hand side of $(\mathrm{C} 2)$ may be written

$$
\begin{aligned}
& -\frac{g}{\theta_{0}}\left\{\frac{\partial \bar{\theta}}{\partial x}\left(\Psi\left(\bar{\theta}+\theta^{\prime}\right)-\Psi(\bar{\theta})-\Psi^{\prime}(\bar{\theta}) \theta^{\prime}\right)\right. \\
& \left.+\frac{\partial \theta^{\prime}}{\partial x}\left(\Psi\left(\bar{\theta}+\theta^{\prime}\right)-\Psi(\bar{\theta})\right)\right\} \\
& \quad=\frac{\partial}{\partial x}\left\{-\frac{g}{\theta_{0}} \int_{0}^{\theta \prime}[\Psi(\bar{\theta}+\eta)-\Psi(\bar{\theta})] d \eta\right\},
\end{aligned}
$$

while the second term in large braces reduces to

$$
\partial\left(\psi^{\prime}, c_{p} \pi_{0}\right) \theta^{\prime}
$$

Using (C4) and (C5) to simplify (C2) then yields (5.27).

\section{Pseudomomentum}

In this section the finite-amplitude flux (6.21) is derived from the pseudomomentum (6.10). As in the pseudoenergy case we first write 


$$
\begin{aligned}
\frac{D A}{D t}= & \frac{\partial A}{\partial \bar{\theta}} \frac{D \bar{\theta}}{D t}+\frac{\partial A}{\partial \theta^{\prime}} \frac{D \theta^{\prime}}{D t}+\frac{\partial A}{\partial \bar{\omega}} \frac{D \bar{\omega}}{D t} \\
& +\frac{\partial A}{\partial \omega^{\prime}} \frac{D \omega^{\prime}}{D t}+\frac{\partial A}{\partial \rho_{0}} \frac{D \rho_{0}}{D t} \\
= & \left(\frac{\partial A}{\partial \bar{\theta}}-\frac{\partial A}{\partial \theta^{\prime}}\right) \frac{D \bar{\theta}}{D t}+\left(\frac{\partial A}{\partial \bar{\omega}}-\frac{\partial A}{\partial \omega^{\prime}}\right) \frac{D \bar{\omega}}{D t} \\
& -\frac{\partial A}{\partial \omega^{\prime}}\left(\frac{\omega}{\rho_{0}{ }^{2}} \frac{d \rho_{0}}{d z} \frac{\partial \psi^{\prime}}{\partial x}+\frac{g}{\theta_{0}} \frac{\partial \theta^{\prime}}{\partial x}\right)-\frac{\partial A}{\partial \rho_{0}} \frac{1}{\rho_{0}} \frac{d \rho_{0}}{d z} \frac{\partial \psi^{\prime}}{\partial x} .
\end{aligned}
$$

Now, in (C6) we have

$$
\begin{gathered}
\frac{D \bar{\theta}}{D t}=\frac{1}{\rho_{0}} \partial\left(\psi^{\prime}, \bar{\theta}\right), \\
\frac{D \bar{\omega}}{D t}=\frac{1}{\rho_{0}} \partial\left(\psi^{\prime}, \bar{\omega}\right), \\
\frac{\partial A}{\partial \bar{\theta}}=\left(\bar{\omega}+\omega^{\prime}\right)\left[R\left(\bar{\theta}+\theta^{\prime}\right)-R(\bar{\theta})\right]-\bar{\omega} R^{\prime}(\bar{\theta}) \theta^{\prime} \\
-\rho_{0}\left[S\left(\bar{\theta}+\theta^{\prime}\right)-S(\bar{\theta})-S^{\prime}(\bar{\theta}) \theta^{\prime}\right], \\
\frac{\partial A}{\partial \theta^{\prime}}=\left(\bar{\omega}+\omega^{\prime}\right)\left[R\left(\bar{\theta}+\theta^{\prime}\right)-R(\bar{\theta})\right]+R(\bar{\theta}) \omega^{\prime} \\
\frac{\partial A}{\partial \bar{\omega}}=\int_{0}^{\theta^{\prime}}[R(\bar{\theta}+\eta)-R(\bar{\theta})] d \eta, \\
\frac{\partial A}{\partial \omega^{\prime}}=\int_{0}^{\theta^{\prime}} R(\bar{\theta}+\eta) d \eta, \\
\frac{\partial A}{\partial \rho_{0}}=-\int_{0}^{\theta^{\prime}}[S(\bar{\theta}+\eta)-S(\bar{\theta})] d \eta .
\end{gathered}
$$

Putting everything together then yields

$$
\frac{\partial A}{\partial t}=-\partial\left(\psi, \frac{A}{\rho_{0}}\right)-\frac{g}{\theta_{0}} \frac{\partial \theta^{\prime}}{\partial x} \int_{0}^{\theta^{\prime}} R(\bar{\theta}+\eta) d \eta+\omega^{\prime} \frac{\partial \psi^{\prime}}{\partial x} .
$$

Finally, the second term on the right-hand side of (C7). may be written

$$
\frac{\partial}{\partial x}\left\{-\frac{g}{\theta_{0}} \int_{0}^{\theta^{\prime}}\left\{\int_{\bar{\theta}}^{\bar{\theta}+\eta} R(\xi) d \xi\right\} d \eta\right\}
$$

while the last term $\omega^{\prime} \partial_{x} \psi^{\prime}$ may be written in the form (6.18); this then yields $(6.21)$.

\section{REFERENCES}

Abarbanel, H. D. I., D. D. Holm, J. E. Marsden; and T. Ratiu, 1986: Nonlinear stability analysis of stratified fluid equilibria. Phil. Trans. R. Soc. London, A318, 349-409.

Abraham, R., and J. E. Marsden, 1978: Foundations of Mechanics. 2d ed. Benjamin-Cummings, $806 \mathrm{pp}$.
Acheson, D. J., 1976: On over-reflexion. J. Fluid Mech., 77, 433472.

Andrews, D. G., 1980: On the mean motion induced by transient inertio-gravity waves. Pure Appl. Geophys., 118, 177-188.

_ 1981: A note on potential energy density in a stratified compressible fluid. J. Fluid Mech., 107, 227-236.

- 1983: A conservation law for small-amplitude quasi-geostrophic disturbances on a zonally asymmetric basic flow. J. Atmos. Sci., 40, 85-90.

- and M. E. McIntyre, 1976: Planetary waves in horizontal and vertical shear: The generalized Eliassen-Palm relation and the mean zonal acceleration. J. Atmos. Sci., 33, 2031-2048.

— Charney-Drazin theorems for waves on axisymmetric mean flows in compressible atmospheres. J. Atmos. Sci., 35, 175-185.

$\longrightarrow$, and - 1978b: On wave-action and its relatives. J. Fluid Mech., 89, 647-664; 1984: Corrigenda. 95, p. 796.

- J. R. Holton, and C. B. Leovy, 1987: Middle Atmosphere Dynamics. Academic Press, 489 pp.

Arnol'd, V. I., 1978: Mathematical Methods of Classical Mechanics. Springer-Verlag, $462 \mathrm{pp}$.

Batchelor, G. K., 1953: The condition for dynamical similarity of motions of a frictionless perfect-gas atmosphere. Quart. J. Roy. Meteor. Soc., 79, 224-235.

Benjamin, T. B., 1984: Impulse, flow force, and variational principles. IMA J. Appl. Math., 32, 3-68.

- 1986: On the Boussinesq model for two-dimensional wave motions in heterogeneous fluids. J. Fluid Mech., 165, 445-474.

Bretherton, F. P., 1969: On the mean motion induced by internal gravity waves. J. Fluid Mech., 36, 785-803.

- 1971: The general linearised theory of wave propagation. Lect. Appl. Math., 13, Amer. Math. Soc., 61-102.

—, and C. J. R. Garrett, 1968: Wave trains in inhomogeneous moving media. Proc. R. Soc. London, A302, 529-554.

Cairns, R. A., 1979: The role of negative energy waves in some instabilities of parallel flows. $J$. Fluid Mech., 92, 1-14.

Garrett, C. J. R., 1968: On the interaction between internal gravity waves and a shear flow. J. Fluid Mech., 34, 711-720.

Grimshaw, R., 1975: Internal gravity waves and their interaction with the mean wind. J. Atmos. Sci., 32, 1779-1793.

— 1984: Wave action and wave-mean flow interaction, with application to stratified shear flows. Ann. Rev. Fluid Mech., 16, 11-44.

Goldstein, H., 1980: Classical Mechanics. 2d ed. Addison-Wesley, $672 \mathrm{pp}$.

Hayashi, Y.-Y., and W. R. Young, 1987: Stable and unstable shear modes of rotating parallel flows in shallow water. J. Fluid Mech., 184, 477-504.

Haynes, P. H., 1988: Forced, dissipative generalizations of finiteamplitude wave-activity conservation relations for zonal and nonzonal basic flows. J. Atmos. Sci. 45, 2352-2362.

Held, I. M., and B. J. Hoskins, 1985: Large-scale eddies and the general circulation of the troposphere. Adv. Geophys., 28A, 331.

Holliday, D., and M. E. McIntyre, 1981: On potential energy density in an incompressible, stratified fluid. J. Fluid Mech., 107, 221225.

Hoskins, B. J., 1983: Modelling of the transient eddies and their feedback on the mean flow. Large-Scale Dynamical Processes in the Atmosphere, B. J. Hoskins and R. P. Pearce, Eds., Academic Press, 169-199.

Killworth, P. D., and M. E. McIntyre, 1985: Do Rossby-wave critical layers absorb, reflect or over-reflect? J. Fluid Mech., 161, 449492.

Lewis, D., J. E. Marsden, R. Montgomery, and T. Ratiu, 1986: The Hamiltonian structure for dynamic free boundary problems. Physica, 18D, 391-404.

Lipps, F. B., 1990: On the anelastic approximation for deep convection. J. Atmos. Sci., 47, 1794-1798.

, and R. S. Hemler, 1982: A scale analysis of deep moist convection and some related numerical calculations. J. Atmos. Sci., 39, 2192-2210. 
Long, R. R., 1953: Some aspects of the flow of stratified fluids. 1. A theoretical investigation. Tellus, 5, 42-57.

MacKay, R. S., and P. G. Saffman, 1986: Stability of water waves. Proc. R. Soc. London, A406, 115-125.

Marsden, J. E., and A. Weinstein, 1983: Coadjoint orbits, vortices, and Clebsch variables for incompressible fluids. Physica, 7D, 305-323.

McIntyre, M. E., 1973: Mean motions and impulse of a guided internal gravity wave packet. J. Fluid Mech., 60, 801-811.

,- 1977: Wave transport in stratified, rotating fluids. Problems of Stellar Convection, E. A. Spiegel and J. P. Zahn, Eds., Springer Lecture Notes in Physics, Vol. 71, 290-314.

- and T. G. Shepherd, 1987: An exact local conservation theorem for finite-amplitude disturbances to non-parallel shear flows, with remarks on Hamiltonian structure and on Arnol'd's stability theorems. J. Fluid Mech., 181, 527-565.

Morrison, P. J., 1982: Poisson brackets for fluids and plasmas. Mathematical Methods in Hydrodynamics and Integrability in Dynamical Systems, M. Tabor and Y. M. Treve, Eds., Amer. Inst. Phys. Conf. Proc., Vol. 88, 13-46.

Ogura, Y., and J. G. Charney, 1962: A numerical model of thermal convection in the atmosphere. Proc. Int. Symp. on Numerical Weather Prediction, Tokyo, S. Syono, Ed., Meteor. Soc. Japan, 431-451.

- , and N. A. Phillips, 1962: Scale analysis of deep and shallow convection in the atmosphere. J. Atmos. Sci., 19, 173-179.

Olver, P. J., 1986: Applications of Lie Groups to Differential Equations. Springer-Verlag, $497 \mathrm{pp}$.
Ripa, P., 1981: Symmetries and conservation laws for internal gravity waves. Nonlinear Properties of Internal Waves, B. J. West, Ed., Amer. Inst. Phys. Conf. Proc., Vol. 76, 281-306.

- 1990: Positive, negative and zero wave energy and the flow stability problem, in the Eulerian and Lagrangian-Eulerian descriptions. Pure Appl. Geophys., 133, 447-466.

Salmon, R., 1988a: Hamiltonian fluid mechanics. Ann. Rev. Fluid Mech., 20, 225-256.

- 1988b: Semigeostrophic theory as a Dirac-bracket projection. J. Fluid Mech., 196, 345-358.

Scinocca, J. F., 1991: Orographically Forced Internal-Wave Instability. Ph.D. thesis, University of Toronto, $165 \mathrm{pp}$.

Shepherd, T. G., 1990: Symmetries, conservation laws, and Hamiltonian structure in geophysical fluid dynamics. $A d v$. Geophys., 32, 287-338.

Vladimirov, V. A., 1987: Integrals of two-dimensional motions of a perfect incompressible fluid of nonuniform density (English transl.). Fluid Dyn., 22, 340-343.

Whitham, G. B., 1965: A general approach to linear and non-linear dispersive waves using a Lagrangian. J. Fluid Mech., 22, 273283.

Wilhelmson, R., and Y. Ogura, 1972: The pressure perturbation and the numerical modeling of a cloud. J. Atmos. Sci., 29, 12951307.

Young, W. R., and P. B. Rhines, 1980: Rossby wave action, enstrophy and energy in forced mean flows. Geophys. Astrophys. Fluid Dyn., 15, 39-52. 